\title{
LE RÉGIME JURIDIQUE DE NAVIGATION DANS LE BASSIN DU FLEUVE CONGO: ENTRE CENTRALISATION, APPLICATION ET REJET D'UN CADRE CONVENTIONNEL RÉGIONAL MAL NÉGOCIÉ EN RÉPUBLIQUE DÉMOCRATIQUE DU CONGO.
}

\author{
Par Camille NGOMA KHUABI*
}

Résumé

La navigation fluviale, lacustre ou maritime constitue un canal important par lequel les pays reliés par un réseau des cours d'eau assurent les échanges commerciaux entre-eux. En Afrique centrale, elle est au cœur des enjeux du développement économique pour les États enclavés comme ceux du bassin du fleuve Congo. Des tentatives de réglementation ont été initiées dès les premières années de la colonisation. Pour les mêmes nécessités, les États réunis au sein de la Commission Internationale du bassin du Congo-Oubangui-Sangha (CICOS)ont inscrit la question au premier chef de leurs préoccupations et ont élaboré un instrument unique, le code de navigation pour la région CEMAC-RDC. Cet article analyse le cadre juridique régional prévu par ces deux instruments. Il démontre que, loin d'être appliqué, ce cadre juridique est ignoré et rejeté par la République démocratique du Congo sur fond d'une souveraineté nationale que ce pays semble revendiquer sur la partie du fleuve qui coule sur son territoire.

\section{INTRODUCTION}

Depuis des temps immémoriaux, fleuves et lacs ont été utilisés pour satisfaire divers besoins humains. Réserves des protéines, ils ont fourni à de nombreuses populations les moyens de survie ; voies de communication, les cours d'eau ont été parcourus par toutes sortes d'embarcations transportant hommes et marchandises. Des règles étaient alors nécessaires pour régenter un secteur dont les activités pourraient mettre en conflits les intérêts des États. La navigation sur les cours d'eau internationaux dût être en ce sens, au cœur de premières conventions internationales élaborées au début du $19^{\text {ème }}$ siècle. Les Actes du Congrès de Vienne du 9 juin $1815,{ }^{1}$ fixant les principes de liberté et d'égalité de traitement

* Assistant et doctorant à la faculté de droit de l'Université de Kinshasa, coordonnateur du CIDHE/ Abl.

1 Charles Rousseau, Précis de droit international public approfondi, Dalloz, Paris, 1975, pp.190-191. Le régime juridique défini par l'Acte de Vienne était d'une application générale traduite graduellement dans la pratique par la conclusion des conventions particulières qui se sont échelonnées au 
à l'occasion de la navigation sur les fleuves internationaux et la Convention de Barcelone du 10 mars $1921^{2}$ sur le statut et le régime des voies navigables d'intérêt international, issue des travaux de la conférence de la Paix de $1919,{ }^{3}$ demeurent les ancêtres d'un « noyau dur $\mathrm{du}$ droit international de l'eau ${ }^{4} \gg$ selon l'expression du professeur Alain pellet. Une toute récente convention adoptée sous les auspices de l'ONU en $1997^{5}$, traite, elle, de l'utilisation des fleuves internationaux à des fins autres que la navigation. Elle met l'accent sur le principe d'une utilisation équitable et raisonnable d'un cours d'eau partagé. Elle privilégie une gestion concertée des ressources naturelles intéressant plusieurs États et précise les facteurs à prendre en considération (géographiques, économiques, sociaux, conservation et protec-

cours de du XIXème siècle. Les plus importantes sont : 1) pour l'Elbe, acte de Dresde (23 juin 1821); 2) pour le Rhin, conventions de Mayence (31 mars 1831) et de Nannheim (17 octobre 1868); 3) pour l'Escaut et la Meuse, le traité de Londres du 19 avril 1839; 4) pour le Danube, traité de Paris du 30 mars 1856, de Berlin du 13 juillet 1878 et de Londres du 10 mars 1883; 5) pour le Congo et le Niger, convention de Berlin du 26 février 1885. La caractéristique essentielle de l'évolution du droit fluvial international au XIXème siècle, c'est la tendance à une internationalisation croissance. Celleci s'affirmation dans deux directions : a) égalité de traitement dans l'exercice de la navigation entre riverains et non-riverains; b) gestion de la voie d'eau par un organisme international, la Commission fluviale. (Idem, p.191).

2 Le statut de Barcelone obéissait à deux principes fondamentaux : a) liberté de navigation, c'est-àdire, libre utilisation du fleuve comme voie de communication : c'est le principe traditionnel; b) égalité de traitement (art. 3 et 4 du statut), corollaire moderne du principe précédent qui implique toute interdiction de toute discrimination entre États contractants au point de vue de l'exercice de la navigation (prohibition de l'établissement des tarifs différentiels suivant la provenance ou la destination des marchandises transportées). Idem, pp. 192-193. Par ailleurs, au critère politique de la définition d'un fleuve international lié aux territoires des États séparés ou traversés, la Convention de Barcelone y superpose un second critère de nature économique : la navigabilité. La convention substitue ainsi à la dénomination traditionnelle de « fleuve international » celle de " voie d'eau internationale » qui permet d'englober les affluents navigables. Elle admet la licéité des taxes rémunérant des services rendus et précise les obligations qui pèsent sur les États riverains, mais laisse le choix des modes de gestion. (Ngyuen Quoc Dinh, Patrick Daillier, Matthias Forteau, Alain Pellet, Droit international public, $8^{\text {ème }}$ édition, L.G.D.J, Paris, 2009, nº713, p.1369; Nguya-Ndila Malengana, Droit international public. Domaine public international, p.49); David Ruzié, Droit international

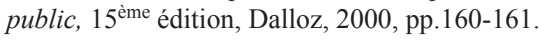

3 Au point de vue du droit fluvial, la conférence de la Paix de 1919 avait une double tâche à remplir : a) fixer le régime particulier des fleuves internationaux intéressant les États ex-ennemis (Rhin, Elbe, Oder, Niémen et Danube). Le régime a été établi par des clauses insérées dans le traité de Paix de 1919 (art. 332 à 337 du traité de Versailles); b) élaborer un régime général applicable à tous les fleuves internationaux, une sorte d'acte de Vienne révisé; prévu par l'article 338 du traité de Versailles. Ce régime devrait par la suite être élaboré par une conférence spéciale convoquée à cet effet par la S.D.N regroupant les États à Barcelone en 1921, idem.

4 Alain Pellet, «Conclusions générales», Société Française pour le droit international, L'eau en droit international, Colloque d'Orléans, Paris, Pedone, 2011, p.396.

5 La convention des Nations Unies sur le droit relatif aux utilisations des cours d'eau à des fins autres que la navigation, New York, le 21 mai 1997, Résolution 51/229 de l'Assemblée générale, annexe, Documents officiels de l'Assemblée générale, cinquante et unième session, Supplément $n^{\circ} 49$ (A) 51/49), pp.286-305. 
tion) et constitue une source d'inspiration pour les États qui veulent se lier dans des accords bilatéraux ou régionaux de coopération fluviale.

En effet, le fleuve Congo avec son vaste réseau navigable s'est déjà révélé important pour le transport, à une époque où la traite négrière était encore un symbole de puissance pour les États qui participaient à cette entreprise criminelle dénoncée encore aujourd'hui. L'arrivée des portugais marchands vers la fin du quinzième siècle, fait entrer l'Afrique centrale dans les échanges intercontinentaux. Avec la traite négrière, un axe commercial va se développer vers le seizième siècle le long du fleuve Congo, de la côte atlantique jusqu'au delà de son cours moyen, avec comme plaque tournante, le Pool Malebo ${ }^{6}$.

En vue de faciliter la liberté de commerce dans l'espace bordé par ce réseau fluvial, l'Acte général de Berlin de 1885 élaboré pour le besoin de la cause, internationalise le fleuve Congo et y impose les règles tirées du droit international européen, consacrées dans les deux premiers textes pré-rappelés. La nécessité d'assurer un service optimal de transport conduit la France et la Belgique à signer la Convention Franco-belge du 11 janvier 1932 relative au balisage du Congo et de l'Oubangui. Parallèlement, une Agence des transports fluviaux commune au Congo Brazzaville, au Tchad et à la RCA fut créée dans le cadre de l'AEF.

Une fois indépendants, les mêmes nécessités vont apparaître pour les États sortis de la colonisation. En dépit de quelques tensions entre riverains, trois États riverains du fleuve, la RDC, la République du Congo et la Centrafrique (RCA) vont ainsi s'engager, en 1978 dans un Protocole d'Accord Tripartite (du 21 juillet 1978) relatif aux modalités d'entretien des voies navigables d'intérêt commun. En 1999, après une longue période de léthargie, l'idée de la création d'un organe de coopération autour du bassin est relancée et aboutit à la création de la CICOS dont les objectifs sont entre autres, d'instituer un régime fluvial uniforme de navigation sur la base du principe de liberté et d'égalité de traitement et d'aménager et d'exploiter les voies navigables sur la base du droit à une participation équitable et raisonnable aux avantages tirés de l'utilisation durable des eaux ${ }^{7}$.

La signature de l'Accord créant cet organisme a coïncidé avec la signature d'un instrument spécifiquement dédié à la navigation intérieure entre riverains : le Code de la navigation intérieure CEMAC-RDC. Ce Code institue un régime juridique applicable à tous les bâtiments et menues embarcations immatriculées dans les territoires de la Communauté Économique et Monétaire de l'Afrique Centrale (CEMAC) et de la République démocratique du Congo $[\ldots]$ en application des conventions internationales en vigueur ${ }^{8}$. Ce cadre juridique commun semble connaître des entraves quant à son application en RDC. Le secteur de navigation peine ainsi à s'affirmer comme moteur de développement et d'intégra-

6 Sylvie AYIMPAM, Vie matérielle, échanges et capitalisme sur la rive méridionale du Pool du fleuve Congo 1815-1930, Collection Clio en Afrique, $\mathrm{N}^{\circ}$ 18, Edition du Centre d'Études des Mondes Africains, Ex-en-Province, 2006, p.5.

7 Article 2 de l'Accord instituant un régime fluvial uniforme et créant la CICOS.

8 Article $1^{\text {er }}$ du Code de la navigation intérieure CEMAC-RDC, N'DJAMENA, 17 décembre 1999, p. 11. 
tion économique, par le potentiel des échanges commerciaux qu'il devrait permettre de drainer, d'abord sur le plan intérieur, ensuite avec l'ensemble des États de la région.

Dans une démarche positiviste, cette étude se propose d'analyser le cadre juridique applicable à navigation sur le réseau fluvial du bassin du Congo en relevant l'état de sa mise en œuvre en République démocratique du Congo (II). Mais, les éccueils liés à son application ne peuvent correctement être évalués qu'après avoir mis en exergue l'étendue du réseau navigable et de ses interconnections qu'il convient de présenter dans une démarche descriptive (I).

\section{RÉSEAU NAVIGABLE DANS LE BASSIN DU FLEUVE CONGO ET INTERCONNEXIONS POSSIBLES}

L'étude de la coopération transfrontalière dans le bassin du Congo relève en effet, que non seulement la navigation a constitué historiquement l'unique objet d'intérêt ayant conduit aux premiers efforts de coopération, mais que son importance a amené les États riverains à multiplier les tentatives d'intégration régionale, malgré des échecs répétés. Pour bien comprendre cet état de choses, il est nécessaire de présenter le réseau des voies navigables (A) dont les caractéristiques géopolitiques rendent parfaitement compte de l'enjeu qu'il représente, au-delà même des frontières de l'ensemble du bassin hydrographique, avant d'en examiner le cadre institutionnel sur le plan strictement interne de la RDC (B).

\section{A. Étendue du réseau de navigation intérieure, caractéristiques et défis du secteur}

Le bassin du Congo s'étend sur environ $3700000 \mathrm{~km} 2$ et compte plus de $15000 \mathrm{~km}$ de voies navigables, ${ }^{9}$ la plus importante étant le fleuve Congo lui-même. Néanmoins, l'existence de nombreuses chutes entraîne la rupture du fleuve en plusieurs biefs et explique son découpage en trois grands tronçons : il y a tout d'abord le cours inférieur constitué par le bief maritime, navigable de l'embouchure à Matadi; ensuite, le cours moyen dans lequel le fleuve redevient navigable sur près de 1740 km entre Brazzaville-Kinshasa et Kisangani; enfin, le cours supérieur. Ce réseau intérieur navigable (1) ouvre le pays à des interconnexions au-delà de l'espace sous régional de l'Afrique centrale compte tenu de la position géographique du fleuve Congo et ses dépendances (2).

\section{1. Étendue du réseau navigable intérieur de la $R D C$}

La RDC possède un réseau de $16238 \mathrm{~km}$ de voies navigables et 40 ports fluviaux notamment sur le fleuve Congo et ses rivières, divisé par deux séries de chutes en trois biefs navigables déjà cités. Le trafic fluvial est particulièrement important dans les provinces intérieures, où l'habitat est essentiellement concentré le long des cours d'eau et où les voies

9 CICOS/GTZ, Plan d'action stratégique pour la promotion de la navigation dans le bassin CongoOubangui-Sangha, Kinshasa, CICOS, 2007 p. 1 (ci-après PAS navigation). 
navigables constituent souvent la seule desserte et y jouent de ce fait, un rôle économique et social de première importance.

Le bief inférieur est constitué des $148 \mathrm{~km}$ que parcourent les grands navires de mer dans le bief maritime du fleuve Congo jusqu'à Matadi. À partir de Matadi, le fleuve devient non navigable jusqu'à Kinshasa. Cette partie du fleuve, impropre pour l'instant à toute navigation, est court-circuitée par un chemin de fer à voie métrique de $365 \mathrm{~km}$ de longueur. Mais, selon Robert Arnould qui s'appuie sur l'exemple des aménagements multifonctionnels de quelques grands cours d'eau tels que le Rhône, le Tennessee et le Yang-Tze-Kiang, une canalisation du fleuve Congo à l'aval de Kinshasa pourrait rendre navigable cette partie du fleuve. L'auteur pense que l'aménagement du Bas-Congo doit être pensé, non seulement en fonction de la production d'énergie hydroélectrique, mais aussi en fonction d'une possible navigation fluviale à grand gabarit entre Kinshasa et l'Océan Atlantique ${ }^{10}$. Le bief supérieur se situe dans le Lualaba au Katanga. Il est caractérisé par un très faible niveau de desserte des zones critiques de la Région de Grands Lacs consécutifs notamment aux insuffisances des institutions de gestion concertée de la navigation sur ce bief.

Le bief Moyen est, lui, constitué de l'ensemble des trois axes principaux Oubangui et affluents ; Kisangani-Kinshasa/Brazzaville et affluents et, Ilebo-Kinshasa/Brazzaville et affluents. Long de 1734 kilomètres entre Kinshasa et Kisangani, il constitue le plus vaste réseau navigable du fleuve Congo sur lequel porte l'essentiel des Accords de coopération qui lie la RDC à ses voisins. Dans l'axe principal d'Oubangui et affluents s'observe une tendance décroissante persistante et alarmante des débits d'étiage et une exposition croissante aux risques de pollution. Ces problèmes de bas niveaux d'eau, en particulier sur l'Oubangui, ont davantage interrompu la navigation depuis les années soixante-dix au cours desquelles les tendances des précipitations indiquaient une diminution de la moyenne annuelle entre 1970 et 2000 (NASA 2010). Navigable jadis pendant huit mois, la navigation n'y est actuellement possible que pendant quatre à cinq mois ${ }^{11}$. Une des réponses proposées pour réduire ce déficit, c'est le transfert interbassin de l'eau, du bassin du Congo au bassin du lac Tchad. Les initiateurs de ce projet citent «la navigabilité permanente de l'Oubangui » comme l'un des bénéfices apportés par un projet qui inclurait un barrage sur ce cours d'eau (près de Palambo, en République Centrafricaine). Ce barrage équilibrerait le flux du fleuve, et réduirait le nombre de jours de niveau d'eau trop faible pour permettre aux vaisseaux de naviguer ${ }^{12}$.

En dépit de l'importance de ce bief, la navigation sur son tronçon fait face à de nombreuses difficultés liées notamment à la non mise en application du code commun de la navigation sur les voies navigables de la CICOS, la non exécution convenable des travaux de balisage, la non exécution convenable des travaux de dragage et de renflouage des épaves,

10 Robert Arnould, La canalisation du fleuve Congo à l'aval de Kinshasa : un défi pour le XXIème siècle, Bulletin de la Société géographique de Liège, n 46, 2005, p.99, Réf. pp.99-117.

11 Entretien avec responsable de la CICOS à Kinshasa ce 21 mars 2014.

12 Anonyme, Défis et opportunités liés à l'eau 3, s.d, p.42; pp.37-122. 
la non prise en considération d'autres solutions visant à améliorer la navigabilité des voies navigables, la non disponibilité des cartes CICOS nautiques de navigation, la non harmonisation des systèmes de références géodésiques $(\mathrm{x}, \mathrm{y}, \mathrm{z})$ du réseau de navigation de la CICOS, le faible niveau de desserte (désenclavement et intégration) des zones critiques de la Région des Grands Lacs consécutif à l'exploration insuffisante des possibilités de desserte fluviale de ces zones et l'insuffisance des équipements d'intervention de la $\operatorname{CICOS}^{13}$. À cesujet, une récente étude du NEPAD ${ }^{14}$ sur les écarts de développement des infrastructures des voies navigables intérieures soulignait que le développement des voies navigables intérieures nécessite la mise en place d'un cadre réglementaire pour assurer un niveau approprié de sécurité conforme aux normes ${ }^{15}$.

Dans le cas de la navigation sur le fleuve Congo, le cadre juridique commun actuellement en construction ne semble concerner que les États signataires d'un Accord encore non appliqué. Ses règles devraient s'étendre à l'ensemble des interconnexions entre les voies navigables et différents corridors de transport appartenant à des sous régions différentes.

\section{Interconnexions entre la navigabilité du fleuve Congo et les différents corridors de transport de la région des grands lacs}

La RDC appartient à trois zones géographiques africaines: l'Afrique centrale comportant l'espace CEMAC; la région des Grands Lacs et la SADC. Les trois régions sont directement reliées par le fleuve Congo à travers ses affluents. Si actuellement la coopération pour la navigation sur le fleuve Congo ne semble concerner que quelques pays de la première région, l'ensemble de pays des deux autres régions pensent réaliser une coopération qui doit nécessairement inclure le réseau fluvial, lacustre et maritime de la RDC. Pour les pays de la SADC, l'objectif poursuivi dans le domaine des transports maritimes et fluviaux est de progresser vers une politique harmonisée et de parvenir en commun, à une vision des avantages nets d'une politique commune du transport et des ports, avec la possibilité d'effets de redistribution dans les États membres. La coopération devra aboutir à la mise au point de normes communes en matière de travaux hydrographiques, de cartographie, de normes de construction navale, de conditions de vie du marin, de protection de l'environnement, de communications à la mer et de formation du personnel ${ }^{16}$.

Pour ceux des Grands Lacs dans lesquels appartient également la RDC, la réalisation d'une véritable coopération entre eux doit passer par l'intégration des systèmes et les voies

13 Conférence Internationale pour la Région des Grands Lacs (CIRGL), Étude de faisabilité sur la réhabilitation et la navigabilité du fleuve Congo, août 2006, p.9.

14 Union africaine, Étude sur le Programme de Développement des Infrastructures en Afrique (PIDA), Rapport de la Phase 1, Provisoire, mars 2011, p.40.

15 Idem, p.41

16 Jean Grosdidier de Matons, Un recueil des instruments juridiques internationaux, Programme de politiques de transport en Afrique subsaharienne Document de travail SSATP No. 73F, pp. 132-133. 
des transports des différents pays. Ils pensent avec raison que la réhabilitation et la navigabilité du fleuve Congo doivent être conçues en interrelation avec les autres projets d'infrastructures de transport proposés dans le cadre de la Conférence Internationale pour la Région des Grands Lacs, « CIRGL », dont: la route transafricaine Lagos-Mombasa; le réseau routier du Corridor Nord; le Corridor Sud (Projet chemin de fer des Grands Lacs); le Corridor Lobito; le chemin de fer Mombasa-Kisangani ${ }^{17}$.

Ces interconnexions et leur importance dans l'intégration économique des régions sont de plus en plus ressenties par les États qui n'hésitent plus à s'engager à signer des Protocole sur les fleuves internationaux. Aussi, le 25 août 1995, les États de la SADC, à l'exception de l'Angola, ont signé un Protocole sur les systèmes fluviaux communs à plusieurs États au sein de la SADC. Ce protocole est entré en vigueur en 1998. Un protocole révisé a été signé le 7 août 2000 par tous les membres, sauf la $\mathrm{RDC}^{18}$. Le Protocole est basé sur les règles dites d'Helsinki et sur la Convention des Nations Unies sur le droit des utilisations autres que la navigation des fleuves internationaux, qui reprend le concept d'utilisation équitable et raisonnable des cours d'eau. Si ce protocole de la SADC ne concerne pas l'utilisation des cours d'eau à des fins de navigation, dans la région des Grands Lacs, cet aspect occupe une importance particulière en raison de l'enclavement des territoires. Les États ont ainsi identifié quatre contraintes considérées comme les plus urgentes à lever au regard de la situation particulière de la région.

La première particularité résulte du fait que dans cet ensemble régional, la RDC occupe une position géopolitique stratégique: son territoire partage neuf frontières avec les neuf autres pays de la Région. Cette situation, très spécifique devrait constituer un atout régional majeur dans un environnement qui favorise le développement économique et social de la région, notamment dans la perspective de l'émergence des bassins transfrontaliers de développement aux aires périphériques de la RDC. La deuxième particularité découle du fait que parmi les neuf pays qui partagent les frontières avec la RDC, cinq dont la RCA au Nord, l'Ouganda, le Rwanda et le Burundi, à l'Est et la Zambie, au Sud, sont des pays enclavés; la RDC, elle même, vaste de $2.345 .000 \mathrm{~km} 2$, est semi-enclavée. La troisième particularité est que si la double situation d'asphyxie à laquelle est confrontée la zone périphérique de la RDC n'est pas levée, cela peut faire subir la même situation d'asphyxie au noyau central de la Région des Grands Lacs. La quatrième particularité est que, paradoxalement, l'essentiel des ressources économiques de la Région des Grands Lacs est concentré dans cette zone périphérique de ce noyau central : ressources pétrolières et forestières dans la partie Ouest ( enclave de Cabinda, Congo Brazzaville, partie Ouest de la RDC); ressources forestières et minérales, pierres précieuses notamment au Nord de la RCA et au Sud-ouest du Soudan du sud; ressources agricoles à l'Est (Ouganda, Rwanda, Burundi, et

17 Sur les détails sur ces interconnexions lire : Conférence Internationale pour la Région des Grands Lacs (CIRGL), Étude de faisabilité sur la réhabilitation et la navigabilité du fleuve Congo, août 2006, pp.2-5.

18 Jean Grosdidier de Matons, Un recueil des instruments juridiques internationaux, Programme de politiques de transport en Afrique subsaharienne Document de travail SSATP $\mathrm{N}^{\circ} 73 \mathrm{~F}, \mathrm{p} .134$. 
Est de la RDC), véritable grenier de la région confronté au taux le plus élevé de la pression démographique de la Région des Grands Lacs; ressources minérales et pétrolières les plus importantes de la Région des Grands Lacs dans la bande Ouest (cupper belt en Zambie et dans la partie Sud-ouest de la RDC, diamant, pétrole en Angola); et les immenses potentialités économiques du noyau central.

Il résulte de cette analyse succincte que la mise en place d'un réseau d'infrastructures de transport constitue la contrainte la plus urgente à lever dans la Région des Grands Lacs afin de détendre la région de la triple situation d'asphyxie à laquelle sont actuellement confrontées deux parties importantes de son territoire. La navigation sur le fleuve Congo demeure à ce titre un atout majeur dans l'atteinte de cet objectif. Bien qu'actuellement les principaux axes du bief moyen reliant Kinshasa à Kisangani restent navigables, l'absence de dragage pendant les dix dernières années et la détérioration du système de signalisation ont créé un environnement dangereux illustré par la multiplication des accidents ${ }^{19}$, 1'insécurité, les actes de piraterie ${ }^{20}$ et les naufrages dont les chiffres pourraient battre le record mondial en termes des victimes. En effet, selon les dernières statistiques du ministère des Transports, la RDC a enregistré 153 cas de naufrages ${ }^{21}$ déclarés entre 2008 et 2011, soit une

19 Quelques cas de collision sont cités à titre purement indicatif : Le 29 juillet 2011, deux embarcations entrent en collision sur la rivière Ruki et fait 50 morts dans la province de l'Équateur; le 2 mai 2009 une autre collision d'un bateau avec un convoi de trois pirogues, s'est produite sur le fleuve Congo : trois corps avaient pu être repêchés contre de nombreux disparus ; le 7 juillet 2007, collision sur le lac Kivu, les 51 rescapés regagnent Bukavu; lundi le 09 mai 2011, une baleinière et un bateau battant pavillon du Congo-Brazzaville se sont heurtés au chenal du fleuve Congo au niveau de la localité N'kana située à $102 \mathrm{Km}$ en amont de Kinshasa.

20 Le 19 janvier 2013, des hommes armés ont attaqué dans la matinée, le canot rapide Kivu King connu sous le nom de bateau Ihusi sur le lac Kivu aux environs de Kabare au Sud-Kivu. Cette embarcation sur le lac Kivu avait à son bord cinquante-sept passagers à bord en provenance de Goma à destination de Bukavu. Une victime a témoigné que parmi les passagers il y avait quatre personnes qui sont montées à bord du canot rapide depuis Goma avec leurs armes. Arrivés sur le large de Birava en territoire de Kabare vers Ibinja Nord à 30 minutes de Bukavu, ils ont maîtrisé les autres passagers en tirant une balle en l'air. Dans la panique, ils ont ordonné au capitaine d'arrêter le navire et aux passagers d'éteindre les téléphones. Ils ont par la suite fouillé tous les passagers en demandant s'il y avait à bord un agent de la Banque internationale pour l'Afrique au Congo (BIAC). N'ayant pas trouvé l'agent recherché, ils ont extorqué tous les biens des passagers. Quinze minutes après, une barque motorisée est apparue pour récupérer les pirates à bord du bateau Ihusi et a pris la direction du Rwanda, a poursuivi la victime, http://radiookapi.net/actualite/20 13/01/19/sud-kivu-des-hommes-armes-attaquent-navire-sur-le-lackivu/? , consulté le 25 janvier 2013.

21 Il en est ainsi notamment de cas de naufrage ci-après : Naufrage à Maluku le 22 décembre 2012 ; à Bikaka dans l'équateur le 21 novembre 2012; sur le lac Kivu le 28décembre 2011: 8 disparus et 7 rescapés; naufrage d'une pirogue à Kazumba, dans le Kasaï-Occidental le 21 décembre 2011 : 7 morts; Naufrage du MB/Glodi faisant 200 rescapés à Mbandaka, le 20 décembre 2011 ; une baleinière fait naufrage à Masimanimba faisant une quarantaine de morts le 2 octobre, 2009 ; le 14 septembre un naufrage sur le fleuve Congo, à 7 kilomètres d'Ankoro, 17 corps repêchés ; le juin 27 2011, 19 disparus dans un naufrage sur le lac Albert à Mahagi; le 18mai 2011, des joueurs du FC Lubilo ont été noyés dans le lac Tanganyika à Bukavu; Le 14 décembre 2011 une dizaine de disparus parmi les naufragés du lac Édouard ; le 15octobre 2011, 7 personnes disparues dans un nau- 
moyenne de 38 cas par an. Ces naufrages et collisions, ont causé des pertes en vies humaines estimées à 4477 personnes au cours de la même période, soit une moyenne annuelle de 1193 personnes décédées; ce qui représente près de 100 personnes tuées par mois sur les voies navigables du pays ${ }^{22}$.

Cette brève présentation démontre que malgré l'avantage qu'il présente pour le transport des produits alimentaires vers les centres de consommation, l'aménagement et l'exploitation de voies fluviales en RDC ont rarement bénéficié de financement à la hauteur des besoins du secteur. Sur le plan interne de la RDC, des organes techniques s'occupent des travaux de balisage et d'entretien des voies navigables. La récurrence des incidents de navigation évoqués ci-dessus pourrait témoigner de l'inefficacité et des limites d'un cadre institutionnel national archaïque, déliquescent, dépassé et non adapté.

\section{B. Cadre institutionnel de la navigation en $R D C$}

L'étendue du réseau fluvial congolais est gérée par des services techniques qui doivent théoriquement assurer les travaux d'entretien et de balisage des voies navigables selon des compétences déterminées par la loi, cela en relation avec les structures internationales ou des pays voisins. Il s'agit de la Régie des voies fluviales, de la régie des voies maritimes RVM et de l'Office national des Transports (ONATRA). D'autres entreprises comme

frage à Nganda Libaya dans la province de l'Équateur ; le 20septembre 2011, 5 corps repêchés dans un naufrage sur le Lac Tanganyika ; le 12juin 2011, deux personnes mortes par noyade dans la rivière Lowa à Walikale; le 9 mars 2010, une noyade dans le lac Mayindombe ; le 2 mai 2011 un naufrage d'une baleinière sur la rivière Kasaï fait100 personnes portées disparues ; Le 16 juin 2010, quatorze morts, bilan d'un chavirement d'une embarcation sur le lac Moero au bord du village Kashishi à la frontière avec la Zambie ; Le 8 juillet 2010, un mort et seize disparus lors d'un chavirement sur le Lac Kivu à Bukavu; le 30 novembre, 2010 un chavirement sur la rivière Luapula, 8 morts et 10 disparus ; le mai 62010 , une pirogue chavire sur le fleuve Congo et fait deux morts à Kindu ; le 25 avril 2011, une pirogue motorisée chavire sur le lac Kivu, de nombreux disparus ; Le 6 avril 2011 un naufrage sur le lac Édouard fait un disparu et dix tonnes de marchandises perdues ; une baleinière sur la rivière Kasaï en partance pour Nyalongo a pris feu, et 200 personnes ont péri dans la périphérie de la localité de Mbendayi, à près de 100 kilomètres de Tshikapa ; le 23 mars 2014, un naufrage d'un bateau dans le lac Albert en provenance de l'Ouganda fait 251 morts parmi les refugiés congolais. (Statistiques compilées par nous-mêmes sur base des informations disponibles dans le site de la Radio okapi).

22 Pour lutter contre ces catastrophes, le 12 mars 2013, le ministre des Transports et voies de communication, a pris une série de mesures pour assainir et sécuriser le secteur fluvial, maritime et lacustre en République Démocratique du Congo. Il s'agit de la fermeture au trafic de plusieurs ports, Beach et chantier navals sur l'ensemble du territoire national, ainsi que l'interdiction de certaines unités fluviales ou embarcation de naviguer. De même, plus de six cents personnes naviguant sont sanctionnées par le ministre pour manque de qualification professionnelle. 
l'OGEFREM ${ }^{23}$ ou la $\mathrm{CMC}^{24}$ n'interviennent que pour le prélèvement des taxes sur des marchandises transportées par des navires ou embarcations se trouvant dans les ports congolais. Nous passons en revue de manière sommaire les compétences dévolues à ces organes de manière à comprendre de quelle manière leurs compétences pourraient être articulées et coordonnées avec celles des structures régionales prévues dans le cadre de la coopération liant la RDC à ses voisins.

\section{La Régie des voies fluviales (RVF)}

La Régie des voies fluviales est une entreprise publique à caractère commercial et industriel, créée par l'ordonnance-loi 71-004 du 26 janvier 1971, et dotée de la personnalité juridique. Aux termes de l'article 3 de l'Ordonnance no $78-199$ du 5 mai 1978 qui la crée ${ }^{25}$, la Régie a pour activité les voies de navigation intérieure. À cette fin, elle est notamment chargée des études et de l'exécution des travaux de balisage; de l'aménagement et de l'entretien des passes de navigation ainsi que du curage du rivage des ports et de l'établissement des levés et des cartes hydrographiques. Elle peut également effectuer toutes opérations se rattachant directement ou indirectement à son objet. Un cahier des charges édicté par l'Ordonnance $\mathrm{N}^{\circ} 71-308$ du 3 décembre 1971 précise l'étendue de ses activités, délimite son champ d'action et détermine les conditions de réalisation de sa mission.

Aux termes de ce cahier des charges ${ }^{26}$, la Régie est chargée 1) d'exécuter les travaux et opérations de balisage des voies de navigation intérieure, comprenant la pose et l'entretien de feux, bouées, balises, signaux, alignements limitatifs et axiaux et autres repères de navigation; 2) de dragage et l'entretien des passes de navigation ainsi que le curage du rivage des ports fluviaux; 3 ) des sondages, levés hydrographiques et autres études et travaux nécessaires pour assurer la sécurité de la navigation et améliorer la navigabilité; 4) de la surveillance des mouvements des plantes aquatiques et le désherbage des bouées, à l'exclusion de toute entreprise d'éradication et de la publication des renseignements intéressant la navigation sur les voies de navigation intérieure s'étendan ${ }^{27}$ sur l'ensemble des tronçons navi-

23 L'Office de gestion du fret maritime a été créé par l'Ordonnance 80-256 du 2 novembre 1980 (J.O.Z., n²2, 15 novembre 1980, p. 8). Aux termes de l'article 5 de cette ordonnance l'Office a mission de promouvoir, par des mécanismes appropriés ou par des actions spécifiques, la productivité nationale dans le secteur du transport maritime, en ayant constamment en vue la recherche de l'efficacité et de la rentabilité de toute l'opération, depuis le producteur jusqu'au consommateur.

$24 \mathrm{LaCMC}$ a pour objet l'armement maritime et l'exploitation de tous navires de transport par mer. Il peut faire toutes opérations quelconques se rattachant directement ou indirectement à son objet ou qui sont de nature à faciliter la réalisation de celui-ci. (Article 3 de la loi n ${ }^{\circ} 74-026$ du 2 décembre 1974 portant création de la Compagnie maritime zaïroise, J.O.Z., n4, 15 février 1975, p. 124.

25 Ordonnance n78-199 du 5 mai 1978 portant statuts d'une entreprise publique dénommée Régie des voies fluviales, en abrégé «RVF».

26 Article $^{\text {er }}$ de l'Ordonnance $N^{\circ} 71-308$ du 3 décembre 1971 établissant le cahier des charges de la Régie des voies fluviales.

27 Article 2 de l’Ordonnance $\mathrm{N}^{\circ} 71-308$ du 3 décembre 1971, op.cit. 
gables de la RDC couvrant $11559 \mathrm{~km}$ sur les $15000 \mathrm{~km}$ des voies navigables ${ }^{28}$ de la RDC, mais les difficultés que le pays a traversé les dernières décennies a conduit la RVF à concentrer ses efforts sur la voie nationale (Kinshasa-Ilebo), en limitant les actions sur les biefs secondaires, et sur ceux où le trafic congolais est faible ${ }^{29}$. En vue d'assurer la bonne exécution de ses obligations, la Régie pourra, moyennant l'autorisation préalable du ministre chargé des transports, s'attacher la collaboration d'organismes spécialisés par une convention conclue sous réserve d'approbation par le ministre susdit et le ministre des Finances. Les prestations de ces organismes seront effectuées sous le contrôle et la responsabilité de la Régie des voies fluviales ${ }^{30}$.

\section{La Régie des voies maritimes (RVM)}

La Régie des voies maritimes est créée par l'ordonnance-loi 71-003 du 26 janvier 1971. Comme la RFV, la RVM actuellement CVM, est une entreprise publique à caractère technique et commercial, dotée de la personnalité juridique. Elle a pour activité les voies de navigation du bief maritime du fleuve Congo. À cette fin, elle est notamment chargée des études et de l'exécution des travaux de balisage; de l'aménagement et de l'entretien des passes de navigation ainsi que du curage du rivage des ports; de l'établissement des levés et des cartes hydrographiques; du pilotage des bateaux. Elle peut également effectuer toutes opérations se rattachant directement ou indirectement à l'objet mentionné à l'alinéa précédent. ${ }^{31}$ Comme la RVF, la CVM réalise ses missions conformément aux dispositions d'un cahier des charges annexé à l'ordonnance.

En vue de la réalisation de son objet, la Régie est habilitée, moyennant l'approbation du ministre des Transports et voies des Communications, à facturer toutes les prestations fournies à des tiers et à en majorer, le cas échéant, les tarifs. ${ }^{32}$ Comme RVF, la CVM est placée sous la tutelle des départements des Transports et Communications, et du Portefeuille, chacun y intervenant dans la sphère de ses attributions spécifiques. ${ }^{33}$

28 le bief Kinshasa-Kisangani, ses principaux affluents et sous-affluents; le bief Kinshasa-Port-Francqui, ses principaux affluents et sous-affluents; le bief Ubundu-Kindu; le bief Kongolo-MalembaNkulu; le lac Tanganyika et le lac Kivu; les rivages des ports fluviaux, notamment ceux des ports de Kinshasa, Mbandaka, Bumba, Kisangani, Ubundu, Kindu, Port-Francqui, Kalemie et Kalundu.

29 Nguya-Ndila, Document manuscrit, inédit, op.cit., p.22.

30 Article 12 de l'Ordonnance $N^{\circ} 71-308$ du 3 décembre 1971.

31 Article 3 de l'Ordonnance n $78-198$ du 5 mai 1978 portant statuts d'une entreprise publique dénommée Régie des voies maritimes, J.O.Z., n¹0, 15 mai 1978, p. 56, in Code Larcier, Tome III, vol. 1, Édition 2002, p.362.

32 Article 4, al.2 de l'Ordonnance n ${ }^{\circ} 78-198$ du 5 mai 1978 portant statuts d'une entreprise publique dénommée Régie des voies maritimes, op.cit.

33 Article 22 de l'ordonnance nº $78-198$ du 5 mai 1978 portant statuts d'une entreprise publique dénommée Régie des voies maritimes, op.cit. 


\section{La Société Congolaise de Transport et Port (SCTP, ex ONATRA)}

L'ONATRA a été créé par le Décret nº 0051 du 7 novembre 1995 portant création et statuts d'une entreprise publique dénommée Office national des transports, en abrégé «ONATRA». À sa création, l'ONATRA est une entreprise publique à caractère industriel et commercial, dotée de la personnalité juridique. Comme les deux dernières entreprises, l'Office est placé sous la tutelle du ministère ayant les transports dans ses attributions ${ }^{34}$ et reste soumis au régime des Entreprises publiques actuellement en transformation. Aux termes de l'article 3 du Décret qui le crée, l'Office a pour objet : $1^{\circ}$ d'exploiter selon les méthodes industrielles et commerciales, les services de transport par eau, par chemin de fer et par route, les ports et les services accessoires ou connexes qui lui sont confiés par l'État ; $2^{\circ}$ la gestion des chantiers navals de Kinshasa, de Boma, de Mbandaka et d'autres chantiers navals qui lui seraient confiés ; $3^{\circ}$ percevoir à son profit les taxes instituées par l'État pour l'utilisation des ports maritimes et fluviaux dont il assure l'exploitation et $4^{\circ}$, il peut également effectuer toutes opérations se rattachant directement ou indirectement à l'objet mentionné aux alinéas précédents.

En matière d'exploitation portuaire, même si actuellement quelques entreprises privées entrent en concurrence avec l'ONATRA, ce dernier demeure le principal exploitant fluvial et garde si non, un monopole d'exploitation de tous les ports placés sur le fleuve Congo et ses affluents dont les principaux sont, en aval, Kinshasa et, en amont, Kisangani et Ilebo à l'extrémité de la rivière Kasaï, principal affluent du Congo.

Il résulte de ce qui précède que, les mesures relatives à la navigation sur le fleuve et sur le bief maritime de la RDC sont sous la responsabilité de ces trois structures techniques qui doivent y appliquer, dans les limites de leurs attributions, les dispositions de l'Ordonnanceloi n ${ }^{\circ} 66-96$ du 14 mars 1966 portant Code de la navigation fluviale et lacustre ${ }^{35}$ et une série des mesures réglementaires en rapport avec cette question. Si les deux premières s'occupent des travaux d'entretien, notamment le balisage des voies navigables sur leurs tronçons respectifs, l'ONATRA devrait tirer profit du vaste réseau fluvial et lacustre intérieur, dans un contexte général d'enclavement d'un pays en faillite, ruiné par le pillage de ses ressources naturelles. De même, la faiblesse des moyens techniques, humains et financiers quasi congénitale de ces entreprises ont eu pour conséquence, une sous utilisation manifeste des

34 Article 28 du Décret n 0051 du 7 novembre 1995portant création et statuts d'une entreprise publique dénommée l'Office national des transports, en abrégé «O.N.A.T.R.A.», in Code Larcier Tome III, vol. 1, Édition 2002 p.127.

35 Ce code était adopté sans mentionner le régime institué par la Convention de Saint-Germain-enLaye, ni dans le sens de sa confirmation ni quant son éventuelle dénonciation. Et selon le professeur Romain YAKEMTCHOUK, cela n'avait pas facilité l'approche juridique du problème successoral des traités multilatéraux normatifs. V. Romain YAKEMTCHOUK, « Le régime international des voies d'eau africaines », p.493. 
capacités du réseau. La transformation des Entreprises publiques décidées ${ }^{36}$ permettra peutêtre d'y remédier.

Depuis quelques temps, le Gouvernement de la République Démocratique du Congo tente de relever les défis en vue d'améliorer la gouvernance dans ce secteur de transport fluvial. Dans le cadre de l'exécution du Projet de Transport Multimodal cofinancé par le Gouvernement congolais et la Banque Mondiale, il est prévu la prise en charge du volet « transport fluvial » dans sa composante II. Les États généraux des voies navigables organisé du 27 au 29 août 2012 par le Ministère des Transports et Voies de Communication, avec l'appui de la Banque Mondiale s'inscrivent dans cette dynamique. La Commission juridique de ces assises a relevé plusieurs entraves d'ordre intene qui bloquent le secteur. Parmi ces dernières, le manque de suivi et de mise en œuvre des normes, accords et engagements internationaux et régionaux (Corridors, SADC, CICOS) pris par la RDC; l'incohérence entre les textes nationaux et internationaux en la matière; le chevauchement des missions des ministères et services qui gèrent l'exploitation du transport par voie d'eau intérieure. Cette Commission a, en outre, recommandé au Gouvernement d'adapter les textes réglementaires aux conditions actuelles d'administration du secteur et la mise en place d'une réglementation efficace et stricte pour la certification des sociétés d'exploitation fluviales et lacustres et des infrastructures portuaires, etc.

Il nous semble que l'actualisation des textes réglementaires réclamée par la Commission juridique devrait trouver des réponses dans l'application du cadre juridique régional dans lequel s'est librement engagé la République démocratique du Congo. Il reste à analyser ce cadre juridique international, ses implications sur le plan interne des États et tenter de comprendre les raisons du refus de sa mise en œuvre, du moins de son rejet partiel par la République démocratique du Congo.

\section{CADRE JURIDIQUE APPLICABLE AU RÉSEAU NAVIGABLE DU BASSIN DU CONGO}

La description du réseau fluvial et lacustre du bassin du Congo a relevé que $62 \%$ de ce dernier se situe sur le territoire congolais et que, la position géographique de ce pays qui partage des frontières avec neuf voisins, permet des interconnexions de ce réseau au-delà du seul cadre régional de l'Afrique centrale dans lequel la RDC s'engage, avec des hésitations à entretenir une coopération. Or, rendre les échanges plus faciles entre les pays d'une même sous-région ne nécessite pas seulement de disposer d'infrastructures de transport adéquates, ni même d'un secteur professionnel compétent pouvant offrir des services de transport de qualité et à des prix compétitifs. Les unes et les autres ne pourront en effet être mis à contri-

36 Loi $n^{\circ} 08 / 007$ du 7 juillet 2008 portant dispositions générales relatives à la transformation des entreprises publiques, J.O, $49^{\text {ème }}$ Année, Numéro spécial 12 juillet 2008. 
bution efficacement que si le cadre juridique qui gouverne leur exploitation le permet ${ }^{37}$. En matière de navigation, l'évolution du droit fluvial a consacré le passage du monopole à celui de la liberté d'utilisation, le plus souvent dans le cadre d'une gestion internationale, caractérisée par une double tendance à la régionalisation et à la centralisation ${ }^{38}$. Dans le bassin du Congo, ce cadre juridique a évolué au gré de l'évolution historique que les États ont connue. S'il est actuellement encadré par un Accord et un code sur la navigation intérieure, il nous semble important de présenter les étapes de la construction de ce cadre juridique (A) avant d'examiner l'attitude de la RDC face à ce dernier (B) tant il est vrai que, les conditions de son élaboration semblent déterminer les positions souvent prises par ce pays.

\section{A. Construction d'un cadre juridique importé}

La situation géographique du bassin du Congo, partagé entre les différentes puissances coloniales de l'époque, principalement la France et la Belgique, a conduit très tôt ces pays à concevoir les bases d'une coopération pour la gestion du fleuve et de ses affluents. Après leurs indépendances, les jeunes États riverains du bassin, jaloux de leur souveraineté ont du faire face à de nombreuses difficultés pour maintenir une quelconque coopération. Un environnement international né en 1997 après une longue négociation au sein des Nations Unies, et un nouveau contexte politique en RDC au cours de la même année - la prise de pouvoir par l'AFDL - vont finir par rendre possible l'élaboration d'un cadre juridique qui aboutit à la création d'une Commission de navigation regroupant trois riverains du bassin du Congo. Si ce nouveau cadre juridique abroge formellement l'Acte général de Berlin de 1885, il n'en demeure pas moins qu'il s'en est largement inspiré et qu'il s'est plus construit dans le cadre de la CEMAC.

\section{L'Acte Général de Berlin de 1885, un ancêtre de l'Accord créant la CICOS}

Entre 1885 et 1960, date à laquelle la plupart d'États de la région du bassin du Congo accèdent à l'indépendance, la navigation sur le fleuve Congo reposait sur l'Acte général de Berlin de 1885. Les Nations signataires y ont exprimé clairement leur volonté et leur intention de coopérer pour une utilisation rationnelle des eaux partagées. Cet instrument posait le principe de la liberté et de l'égalité des États en ce qui concerne la navigation sur le fleuve Congo. Il s'agissait là des principes conventionnellement appliqués à des fleuves de l'Europe et de l'Amérique, et notamment au Danube, avec les modifications prévues par les traités de Paris de 1856, de Berlin de 1878 et de Londres de 1871 et de $1883^{39}$.

Ce texte fut modifié par des instruments ultérieurs. D'abord, l'Acte général de la conférence de Bruxelles du 2 février 1890 avait renforcé le régime de la répression du commerce

37 Marc H. Juhel, Préface, in Jean Grosdidier de Matons, Un recueil des instruments juridiques internationaux, mai 2004, p. xiii.

38 David Ruzié, Droit international public, $15^{\mathrm{ème}}$ édition, Paris, Dalloz, 2000, p.160.

39 Acte général de la Conférence de Berlin (1885), préambule. 
de l'esclavage condamné déjà à Berlin en 1885; ensuite la Convention de Saint-Germainen-Laye du 10 septembre 1919 a étendu la compétence de l’État territorial en supprimant certaines interdictions imposées par l'Acte général de Berlin et en limitant les interdictions maintenues. L'analyse de ces trois textes démontre en effet que, l'Acte de Berlin avait formellement interdit les droits de douane à l'importation; la Déclaration de Bruxelles avait quant à elle, imposé un plafond très peu élevé de $10 \%$ à l'entrée des marchandises, alors qu'en 1919, la Convention de Saint-Germain-en-Laye (art.2, al. 3) avait carrément reconnu la liberté de fixation des règles et tarifs par l'État territorial. Il s'agissait là, selon le professeur Nguya-ndila ${ }^{40}$, des allègements aux entraves à l'exercice du pouvoir par le souverain territorial. Que devenait alors les règles sur la navigation?

En dépit de ces quelques modifications en faveur de l'État territorial, la liberté de navigation restait garantie par plusieurs prohibitions imposées à ce dernier qui pouvait néanmoins percevoir certaines taxes: aucune entrave ni redevance, aucun péage, maritime ou fluvial, basé sur le seul fait de la navigation, aucun droit de transit sur les marchandises qui se trouvent à bord n'étaient admises. Les seules taxes autorisées sont celles liées à la rétribution pour les services rendus à la navigation (art. 6). De même, on a conservé l'assimilation au régime des fleuves et lacs, les voies artificielles destinées à suppléer à l'innavigabilité. Le régime posé à Berlin avait déjà prévu la création d'une Commission Internationale de Navigation qui devrait assurer la gestion des voies d'eau, mais celle-ci n'ayant jamais fonctionné, la Convention de Saint-Germain-en-Laye est venue combler cette lacune en revenant carrément au droit commun, c'est-à-dire, à la compétence des États riverains qui devenaient ainsi libres de réglementer et de contrôler la navigation sur les tronçons coulant sur chacun de leurs territoires. Mais leurs règlements devraient tendre à faciliter la circulation des navires marchands ${ }^{41}$.

Après les indépendances, ce régime fut dénoncé par Kinshasa qui commence par rebaptiser le fleuve du nom donné à l'État en octobre 1971, et qui réfute par la suite son caractère international devant l'Assemblée générale des Nations unies le 2 novembre au motif que «Sur son parcours de plus de 4000 kilomètres, le fleuve Zaïre [...] ne fait frontière avec un terrain étranger que sur un trajet négligeable de près de 200 kilomètres. C'est donc juridiquement un fleuve intérieur ${ }^{42} »$. Ce qui a été à l'origine des tensions entre le Congo alors Zaïre et son voisin d'en face le Congo Brazzaville. Cet argument fondé sur une «doctrine de supra-riveraineté » selon Mutoy Mubiala ${ }^{43}$ ou sur la doctrine de la souveraineté absolue

40 Célestin NGUYA-NDILA, Indépendance de la République démocratique du Congo et les engagements internationaux antérieurs, publications de l'Université de Kinshasa, 1971, p.42. Pour plus de détails, pp.39-45

41 Article 8 de la Convention de Saint-Germain-en-Laye

42 NATIONS UNIES, Assemblée Générale, Documents officiels de la vingt-sixième session, doc. A/C.6/SR.1273, 2 novembre 1971, p.155.

43 MUBIALA MUTOY, L'évolution du droit des cours d'eau internationaux à la lumière de l'expérience africaine, notamment dans le bassin du Congo/Zaïre. Presses universitaires de France, Paris, 1995. pp.22 à 25 . 
à laquelle on fait parfois référence ou la Doctrine de Harmon ${ }^{44}$ du nom du Procureur Général des États-Unis qui a inventé l'expression en $1895^{45}$ au cours d'un litige sur le Rio Grande, constitue un acte politique qui sera abandonné lorsque les tensions s'apaiseront quelques années plus tard. Parallèlement, d'autres tensions fondées apparemment sur la même doctrine, apparaissent entre la République populaire du Congo et ses autres voisins, lorsque cette dernière décide de nationaliser l'agence des transports fluviaux, alors que celle-ci était jusque-là commune au Congo, au Tchad et à la Centrafrique, par héritage colonial de l'AEF. La doctrine de la souveraineté absolue souvent citée par les riverains en amont considère que les états ont des droits absolus sur l'eau qui traverse leur territoire. La position opposée, également extrême qui est plus favorable aux circonstances des riverains en aval est celle de l'intégrité riveraine absolue, qui protège le cours naturel du réseau fluvial international. Ces positions extrêmes, que sont la souveraineté absolue et l'intégrité riveraine absolue, ont été essentiellement discréditées dans une jurisprudence internationale à la suite de l'affaire du Lac Lanoux portée devant un tribunal arbitral en 1957, qui a soutenu une doctrine de souveraineté territoriale limitée.

Malgré ces tensions très répétées, les États ne renoncent pas à signer des accords. Ainsi, la Centrafrique et le Congo règlent leur différend par un accord en 1970. L'Accord prévoit la création d'une commission mixte pour l'aménagement du fleuve et de certains de ses affluents entre Brazzaville, Bangui et Ouango. La RDC quant à elle, s'entend avec l'Angola en 1978, pour mettre sur pied une Commission pour l'aménagement et l'entretien du bief maritime du fleuve. Enfin, en 1986, ce pays signe avec la Centrafrique un accord pour la construction d'un barrage hydroélectrique sur l'Oubangui, à hauteur de Mobaye. L'effort de coopération le plus marquant durant ces années concerne une réunion qui s'est tenue, du 18 au 23 juillet 1978, à Bangui et qui regroupait l'Empire centrafricain, la République populaire du Congo et la République du Zaïre dans le cadre d'une Commission tripartite pour les transports fluviaux (la présence du Tchad en qualité d'observateur est assurée par un représentant du pays). Envisagée comme un « relais des accords des puissances coloniales sur l'entretien des voies navigables », cette Commission donne lieu à l'adoption de quatre protocoles d'accord ${ }^{46}$ portant sur l'assistance mutuelle des unités fluviales naviguant sur le fleuve Congo/Zaïre et l'Oubangui; les modalités d'entretien des voies navigables d'intérêt commun pour le Congo, le Zaïre et l'Empire Centrafricain; l'élaboration de la législation

44 Illustration de la doctrine de la souveraineté absolue, cette doctrine préconisait qu'en l'absence de législation contraire, les États devaient être libres d'utiliser les ressources en eau de leur juridiction sans tenir compte des effets provoqués au-delà de leurs frontières. Des variantes de cette approche subsistent encore actuellement dans la législation nationale de nombreux pays. Au Kazakhstan, la loi parlementaire de 2001 proclame que toutes les ressources en eau provenant de son territoire lui appartiennent. (Rapport mondial sur le développement humain, 2006, chapitre 6, la gestion des eaux transfrontalières, pp.215-216).

45 Claudia W. Sadoff, David Grey, Les fleuves internationaux d'Afrique Une perspective économique, Banque Mondiale, p.51, http://earthmind.net/rivers/docs/worldbank-fleuve-internationauxd'Afrique.pdf, consulté le 26 août 2013.

46 Idem. 
fluviale commune aux trois États et la création de la Commission de conciliation pour tout différend concernant la navigation fluviale. À la fin de ses travaux, la Commission tripartite demande à la Commission Économique pour l'Afrique (CEA) de procéder à une étude sur l'aménagement global du bassin. Cette étude, réalisée entre 1983 et 1984, portait sur la création d'une organisation intergouvernementale pour l'aménagement et l'exploitation du bassin du fleuve Congo/Zaïre. La majorité des riverains du bassin Congo : l'Angola, le Burundi, la République centrafricaine, la République du Congo, le Rwanda, le Zaïre et la Zambie participent aux réunions et travaux basés sur cette étude. Le régime proposé par l'étude concernait aussi bien la navigation que les autres utilisations, et prévoyait la création d'une autorité constituant un véritable organisme d'intégration économique sous-régionale ». Mais, les experts congolais ${ }^{47}$ qui participaient à l'étude marquaient déjà leur réticence en recommandant que la nouvelle organisation développe une politique qui s'appuie sur les services techniques nationaux et les structures sous-régionales existants, et réciproquement.

Selon Cécile PILARSKI, ${ }^{48}$ l'ambition de ce projet, à travers la participation d'organismes tels que la CEEAC, la CEPGL, le PNUD et l'UDEAC, démontre que de nombreux acteurs, en dehors des riverains, étaient conscients des enjeux présents dans ce bassin. Toutefois, cet effort n'a pas été suivi d'effets et le projet de la Commission économique des Nations unies pour l'Afrique n'a jamais vu le jour. Ce repli s'explique en partie par l'existence de tensions politiques entre les différents riverains; tensions qui aboutiront à la restriction des acteurs dans les nouvelles tentatives de négociation qui contribueront à l'inaction de la Communauté économique des pays des Grands Lacs (CEPGL), un autre organe de coopération transfrontalière entre le Zaïre/RDC, le Burundi et le Rwanda, créée en 1976. ${ }^{49}$ Aussi, en dépit des engagements formels pris par les États dans le cadre de la Commission tripartite de 1978, et malgré l'insistance répétée de la RCA, aucune autre rencontre n'a pu se tenir par la suite.

Ces tentatives de coopération transfrontalière ont eu néanmoins le mérite d'avoir posé les jalons sur lesquels allaient se construire les bases des discussions ultérieures. D'une part, l'échec de la Commission tripartite va servir d'argument pour l'élaboration d'une nouvelle Commission internationale, et d'autre part, c'est grâce à cette réunion que d'importants travaux ont pu être initiés. Ces travaux ont ainsi permis d'envisager pour la première fois l'élaboration d'un organe de coopération autour du bassin, portant sur tous ses usages.

Dans ce contexte, le régime de navigation sur le fleuve Congo devrait continuellement et finalement être régi par les règles posées à Berlin et telles que modifiées par la Déclara-

47 Référence est ici faite à un manuscrit du professeur Célestin NGUYA-NDILA, à propos du projet de création d'une organisation intergouvernementale pour l'aménagement du fleuve du Congo, p. 16.

48 CÉCILE PILARSKI, La gestion partagée des ressources en eau dans le bassin du Congo : état des lieux et perspectives, Mémoire présenté à la Faculté des études supérieures de l'Université Laval, 2009 , p.66.

49 CÉCILE PILARSKI, op.cit., p. 67. 
tion de Bruxelles du 2 juillet 1890 et la Convention de Saint-Germain-en-Laye du 10 septembre 1919, aucun texte contraire n'ayant été pris en remplacement de ceux laissés par les colonisateurs et acceptés par les États nouvellement indépendants, par le biais de l'opération juridique de succession d'États ${ }^{50}$. Mais, devant l'existence des anciens États signataires, le paradoxe théorique qui subsistait était de considérer que la liberté de navigation et de l'égalité de traitement était encore reconnue pour les anciènnes puissances, ce qui pouvait difficilement s'appliquer, les différentes positions prises par les nouveaux États ayant en même temps démontré qu'ils ne s'accordaient pas la même liberté sur les voies navigables.

L'avènement de la CICOS en 1999 devrait finalement mettre un terme à 114 ans d'un régime juridique à l'origine de plusieurs tensions entre les États de la région, et théoriquement régir la navigation sur le bassin du Congo. La RDC semble y voir plutôt un instrument de la CEMAC, une sous-région à laquelle elle n'appartient pas.

\section{L'Accord créant la CICOS et le Code CEMAC-RDC: un nouveau cadre juridique " d'inspiration étrangère » à la $R D C$}

Si l'Acte général de Berlin dénoncé après les indépendances par de simples déclarations de la RDC, était d'inspiration européenne et destiné pour les États européens dans un contexte de colonisation, l'Accord créant la CICOS et le Code CEMAC-RDC sont les deux nouveaux instruments de coopération élaborés sous l'impulsion des États de la CEMAC, riverains du fleuve avec une implication forcée et tardive de la RDC. Nous en examinons tour à tour le contenu avant de tenter de comprendre les raisons qui sous-tendent l'attitude de la $\mathrm{RDC}$ face à ces instruments « régionaux ».

\section{a. L'Accord créant la CICOS et la RDC: un mariage forcé}

L'Accord créant la CICOS et le Code CEMAC-RD ont été élaborés dans le cadre de la CEMAC. Créée en 1959 au moment des indépendances, l'Union douanière et économique, est une organisation d'intégration pour les anciennes colonies de l'AEF qui fonctionnent avec le franc CFA. Cette institution va élargir progressivement ses domaines de compétences ainsi que ses membres, en devenant d'abord l'Union Douanière et Économique de l'Afrique Centrale (UDEAC), en 1964, puis la CEMAC en 1994, mais le remplacement effectif ne se fera toutefois qu'en 1999. Six États sont aujourd'hui membres de la Communauté : le Cameroun, la RCA, la République du Congo, le Tchad, la Guinée Équatoriale et le Gabon. Dans le cadre des objectifs qui lui sont assignés, la CEMAC tend à « harmoniser les réglementations des politiques sectorielles dans les domaines essentiels prescrits par les textes organiques, à savoir l'agriculture, l'élevage, la pêche, l'industrie, le commerce, les transport

50 Pour plus de développements, v. Nguya-ndila (C), Indépendance de la République démocratique du Congo et les engagements internationaux antérieurs, publications de l'Université de Kinshasa, 1971. 
et télécommunications, l'énergie et l'environnement, la recherche, l'enseignement et la formation professionnelle ${ }^{51} »$.

Consciente des problèmes liés au manque d'harmonisation des règles régissant le transport fluvial sur les voies d'eau navigables de ses membres, la CEMAC dépose, à la fin de 1996, une requête auprès de la Commission économique des Nations unies pour l'Afrique (UNCEA) pour le financement d'une étude visant la création d'un code de navigation intérieure des États membres de la CEMAC. L'UNCEA répond favorablement à cette demande, mais pose comme condition la participation du Congo-Kinshasa, celui-ci étant un riverain majeur à l'échelle du bassin ${ }^{52}$. La CEMAC cherchant à rallier la RDC - selon la recommandation de l'UNCEA - devrait élargir, mais en dissimulant, sinon trouver ailleurs, les fondements juridiques devant inspirer l'Accord créant la future Commission ${ }^{53}$. La rédaction du texte doit donc écarter toute référence aux instruments de la CEMAC, si cela n'était pas fait à la demande de la RDC.

En effet, le peu d'attention accordée aux activités autres que la navigation par les pays des continents européen et américain, berceaux du droit des cours d'eau internationaux a conduit à l'établissement d'une règle classique qui consacrait la priorité de la navigation sur toutes les autres utilisations ${ }^{54}$. La Convention de New York du 21 mai 1997 sur le droit relatif aux utilisations des cours d'eau internationaux à des fins autres que la navigation ${ }^{55}$ est, elle, venue réglementer d'autres usages de l'eau non maritime dont le développement, l'attribution et l'utilisation sont en passe de devenir un problème économique, social et politique d'importance majeure. Des auteurs ont même affirmé qu'au seuil du troisième millénaire, des conflits armés pourraient éclater autour de cette ressource ${ }^{56}$. La convention com-

51 CEMAC, Présentation de la CEMAC, [En ligne], [www.cemac.cf/presentationCEMAC.htm]

52 CÉCILE PILARSKI, op.cit., p. 69

53 Selon notre propre analyse faite de la démarche suivie par les autorités de la CEMAC.

54 Institut de droit international, point II/4 de la Résolution sur les usages des cours d'eau internationaux, Annuaire de l'Institut de droit international 1911, vol. 24, p. 365 ; article 8 de la Convention de Genève du 9 décembre 1923 relative à l'aménagement des forces hydrauliques intéressant plusieurs États, Société des Nations, Recueil des traités, vol. 36, p. 75.

55 Résolution A/51/229 du juillet 1997. Le texte de la Convention de New York du 21 mai 1997 est joint en annexe, disponible sur, http://www.un.org/french/documents/view_doc.asp?symbol=A/RE $\mathrm{S} / 51 / 229 \&$ Lang $=\mathrm{F}$

56 Mara TIGNINO, L'eau et son rôle dans la paix et la sécurité internationales, www.icrc.org/fre/ assets/files/other/irrc-879-tignino-fre.pdf ; Jochen Sohnlel, « Le dispositif juridique de l'Europe pour appréhender les conflits transfrontaliers sur l'eau », Lex Electronica, vol. 12 n², 2007 ; HELLENDORFF Bruno, L'eau, les conflits et la coopération. Gestion de l'eau en Afrique de l'Ouest : Risques et opportunités, Note d'Analyse du GRIP, 2013, Bruxelles, http://grip.org/fr/nod e/797, consulté le 30 juillet 2013 ; Bruno Hellendorff, «L'eau, les conflits et la coopération Gestion de l'eau en Afrique de l'Ouest : Risques et opportunités ", in www.grip.org/sites/grip.../ NA_2013-02-08_fr_B-HELLENDORFF.pdf consulté le 13 février 2013,; Frédéric Julien, L'eau dans les relations internationales : la guerre ou la paix? D'un déterminisme à l'autre, (Université d'Ottawa), Congrès AFSP 2009, Section thématique 30 L'hydro politique et les relations internationales http://www.congresafsp2009.fr/sectionsthematiques/st30/st30julien.pdf, le 26 août 2013. 
porte un article 3 consacré aux accords des cours d'eau. Les débats houleux qui ont précédé son adoption justifient ses cinq paragraphes dont le paragraphe 2 qui stipule : «Nonobstant les dispositions du paragraphe 1, les Parties à des accords visés au paragraphe 1 peuvent, si besoin est, envisager de mettre lesdits accords en harmonie avec les principes fondamentaux de la présente Convention. "

Les États riverains du fleuve Congo déjà liés dans des accords bilatéraux de coopération sur certains biefs navigables du réseau du bassin, se sont tout de même appuyés sur les paragraphes 2 et $3^{57}$ de cet article, même si le paragraphe $1^{58}$ de l'article pouvait leur permettre de garder le statu quo. Se fondant sur l'ensemble de ces textes, les États de la CEMAC prirent l'initiative de relancer les débats en vue de créer une Commission après les échecs passés.

Dans une démarche diplomatique subtile ${ }^{59}$, et en marge de la $11^{\text {ème }}$ session de l'UDEAC, la Commission tripartite créée en 1978 fut de nouveau appelée à se réunir, le 24 avril 1998, à Bangui, avec cette fois-ci, la participation du Cameroun. À l'occasion de cette réunion, les experts déplorent l'absence totale de mise en application des protocoles d'accords signés, ainsi que les problèmes persistants qui en découlent en matière d'utilisation et d'entretien des voies d'eau navigables dont ils sont riverains. Constatant ainsi la faiblesse des instruments juridiques existants et désireuses de combler un vide juridique persistant, les parties présentes envisagent la création d'une Commission internationale de navigation. L'UNCEA est à nouveau sollicitée pour le financement d'une étude relative à la création de cette Commission internationale. Accédant une fois de plus à la requête de la CEMAC, l'UNCEA fait appel à un expert de la Commission internationale du Rhin afin de définir les termes de l'accord.

Après la réunion tenue à Kinshasa en avril 1999, au cours de laquelle le projet de code de navigation et celui de Commission ont été amendés, l'Accord instituant un régime fluvial uniforme et créant la Commission Internationale du bassin Congo-Oubangui-Sangha (CICOS) (ci-après l'Accord) est adopté à Brazzaville, le 6 novembre 1999, par les ministres responsables de la navigation. La ratification par les États membres est cependant retardée par les tensions politiques déjà évoquées et la RDC sera le dernier pays à ratifier l'Accord en 2003. Il est de ce fait intéressant d'en analyser le contenu et la portée afin d'apprécier la pratique suivie par la RDC.

57 «Les États du cours d'eau peuvent conclure un ou plusieurs accords, ci-après dénommés "accords de cours d'eau", qui appliquent et adaptent les dispositions de la présente Convention aux caractéristiques et aux utilisations d'un cours d'eau international particulier ou d'une partie d'un tel cours d'eau ».

58 À moins que les États du cours d'eau n'en soient convenus autrement, la présente Convention ne modifie en rien les droits ou obligations résultant pour ces États d'accords en vigueur à la date à laquelle ils sont devenus parties à la présente Convention.

59 C'est nous qui le soulignons. 


\section{aa. Contenu de l'Accord créant la CICOS}

L'Accord instituant un régime fluvial uniforme et créant la CICOS régit les aspects relatifs à la navigation dans les eaux intérieures des États parties. Aux termes de l'article 2 de l'Accord, les États acceptent de : (i) instituer un régime fluvial uniforme de navigation sur la base du principe de liberté et d'égalité de traitement, (ii) d'aménager et d'exploiter les voies navigables sur la base du droit à une participation équitable et raisonnable aux avantages tirés de l'utilisation durable des eaux et de (iii) créer à cet effet une Commission internationale du Bassin Congo-Oubangui-Sangha. Un mandat clair et précis ${ }^{60}$ est confié à cette Commission instituée ${ }^{61}$ : la réalisation des objectifs définis à l'article 2 dans une démarche à court, moyen et long terme. Les objectifs à court terme sont essentiellement d'appliquer les réglementations existantes, de faire la police du trafic fluvial et de mettre en place des normes communes, en particulier en matière de matériels. Les objectifs à moyen terme consistent à formuler et appliquer une politique cohérente d'entretien et une politique de transport susceptible d'ouvrir l'accès aux zones enclavées. Enfin, les objectifs à long terme sont d'étendre l'application de l'Accord à d'autres bassins ainsi qu'aux lacs de la sous-région. La CICOS devrait ainsi mener des négociations devant aboutir à l'élargissement du réseau inter États afin d'y inclure les deux tronçons de la RDC devant le compléter à savoir : le tronçon allant de Kisangani au confluent du fleuve Congo avec l'Oubangui, le tronçon de la rivière Kasaï partant d'Ilebo jusqu'au confluent du fleuve Congo et le réseau lacustre de la RDC (Art. 17).

L'Accord consacre le vieux principe de la liberté de navigation pour toutes les nations (Art. 4), mais il opère une distinction entre État riverain et État contractant (Art. 3). Si l'État contractant est tout partie à l'Accord, l'État riverain est celui dont tout ou partie du territoire est baignée par une voie d'eau du bassin Congo-Oubangui-Sangha. Cette distinction a de l'incidence sur le champ d'application de l'Accord, qui lui, aux termes de l'article 3, ne s'étend dans un premier temps que sur les voies de navigation du bassin Congo-OubanguiSangha situées sur les États contractants, ensuite sur les voies navigables des États contractant, enfin, sur l'ensemble des voies d'eau intérieures déclarées ouvertes à la navigation internationale par la Commission au fur et à mesure des besoins d'intégration.

La formulation de cet article indique que l'Accord devrait être appliqué de façon échelonnée. Son application sur l'ensemble des voies navigables dépendrait donc de travaux à mener éventuellement par la Commission sur des voies non navigables et, de l'acceptation par les États, d'ouvrir à la navigation les voies navigables situées sur leurs territoires selon le principe de liberté et d'égalité de traitement évoqués à l'article 2. Cette formulation visait peut-être à obtenir de la part des États, leur consentement à être lié par l'Accord, mais elle a laissé à ces derniers une large liberté à pouvoir déterminer sur leur territoire, les tronçons d'un cours d'eau du réseau visé par l'Accord, que l'une ou l'autre partie veut ouvrir l'accès 
aux activités des États voisins. L'Accord est ainsi menacé si l'un des États ou un groupe d'États sur les territoires (du) desquels coule le plus important tronçon navigable décide(nt) de ne pas l'ouvrir au profit des autres États contractants. Son application demeure ainsi subordonnée à la volonté de l'un ou l'autre État selon les humeurs de ses dirigeants. De même, l'article 4, alinéa 2 renforce les pouvoirs des États dans la détermination ou le choix des tronçons qu'ils veulent ouvrir à la navigation au profit des autres États. En même temps qu'il proclame le principe de liberté de navigation à son art. 4 al. $1^{62}$, l'alinéa 2 du même article restreint cette liberté pour le transport. En effet, le transport des marchandises et de passagers entre deux points du même État (cabotage national) par tout bateau battant pavillon d'un autre État contractant n'est possible qu'après un accord spécifique entre cet État du pavillon, partie à l'Accord, et celui du tronçon navigable, mais le transport pour le bateau des pays tiers, non partie à l'Accord est lui, soumis à un régime particulier fixé par la Commission. Il reste à savoir si les États riverains peuvent être comptés parmi les États tiers et pourraient dès lors être soumis à ce régime.

Enfin, l'article 6 interdit toute idée d'impôt ou taxe à caractère fiscal pour le seul fait de la navigation, mais permet le prélèvement des frais rémunératoires pour les prestations fournies aux usagers. Le texte cite de manière non exhaustive des droits, taxes et redevances destinés à couvrir d'une manière équitable et raisonnable les dépenses de construction, de maintien et d'amélioration de la voie navigable et de ses accès, ainsi que de la construction d'ouvrages faits dans l'intérêt de la navigation. Si le texte précise que les frais relatifs aux travaux de dragage, de balisage, de construction, d'entretien et le fonctionnement des routes, chemin de fer, canaux latéraux et ouvrages de jonction sont assimilés à de telles dépenses et qu'une sorte de nomenclature des droits et taxe sera rendu public, il ne détermine pas qui est cet usager qui en serait redevable : l'État du pavillon ? Les propriétaires des bateaux et embarcations? Les passagers? L'imprécision des termes utilisés devrait donc être source de plusieurs pratiques décriées dans la région.

Encore une fois, ces genres de contradictions réduisent l'Accord en un simple document de consommation extérieure destinée à conférer des signes de modernité à une époque où la gestion de l'eau, dans tous ses aspects demeure un enjeu mondial ${ }^{63}$. Son contenu traduit bien le climat général qui a prévalu lors de sa négociation, et les conditions dans lesquelles il a été accepté par des États qui, bien qu'abritant sur leur territoire la majeure partie de la matière, en l'espèce, les voies navigables, objet de l'accord, n'auront pas été à la base

62 «La navigation sur les voies navigables visées à l'article 3 est entièrement libre et ouverte aux bateaux de toutes les nations pour le transport de marchandises et de personnes, directement ou avec transbordement, à condition de se conformer aux dispositions contenues dans le présent Accord, notamment les règlements communs édictés en vue d'assurer la sécurité de la navigation sur le Bassin Congo-Oubangui-Sangha»

63 En ce sens, l'Article 15 concluant sur les Dispositions spéciales applicables en cas d'urgence ou de circonstances exceptionnelles (comme un conflit) insiste sur l'importance d'une gestion intégrée du bassin, l'utilisation optimale des voies navigables existantes et sur la communauté d'intérêts des parties à l'Accord. 
de l'initiative de son élaboration. Aussi, ce que des États comme la RDC pouvaient refuser en s'enkystant derrière les notions comme celles de souveraineté absolue ou de supra-riveraineté rappelées plus loin, ont pu l'accepter en obtenant des pouvoirs les plus étendus face au fameux principe de liberté pompeusement proclamée.

En conséquence, si un État comme la RDC venait à décider de refuser d'une part, que la Commission déclare ouvertes ses voies d'eau intérieures dans le sens de l'article 3.3 et d'autre part, que cet État refuse de conclure des accords particuliers avec des États parties pour le transport des marchandises et des passagers, dans le sens de l'article 4.2., l'Accord pourrait voler en éclat ou manifestement en souffrir, du moins en ce qui concerne la navigation. Même si à l'heure actuelle, tel n'est pas encore le cas, la pratique suivie par la RDC semble démontrer que cet État reste encore réticent quant à l'ouverture de son réseau fluvial à la navigation des États parties. Il le serait davantage pour les États tiers dans le lot duquel on doit peut-être classer les États riverains au sens de l'article 1.2 de l'Accord. Dans ces conditions, l'application d'une fameuse clause de la nation la plus favorisée que semble prévoir l'article 5.5 ne serait qu'un leurre. Il reste à rechercher les raisons pouvant justifier l'attitude de la RDC face au régime juridique pourtant accepté.

\section{bb. Les raisons possibles de l'attitude de la RDC face au régime instauré par l'Accord}

L'attitude de la RDC qui peut paraître quelque peu étonnante, résulte des tensions qui entourent la question de la gestion commune des cours d'eau transfrontaliers. En effet, les gouvernements de la plupart des États considèrent que l'eau qui traverse leur pays est une ressource nationale. Par le fait qu'ils traversent sans discrimination des frontières politiques de plusieurs États, la gestion des fleuves devient compliquée en ce sens que nombreux sont les utilisateurs qui doivent se la partager. Aussi, les bassins fluviaux qui sont intégralement à l'intérieur d'une nation suscitent toujours des débats et des discordes, dans une mesure plus ou moins grande, entre utilisateurs ayant des besoins et des préférences de gestion opposées $^{64}$.

Claudia Sadoff et David Grey expliquent que « le contrôle des fleuves et des débits fluviaux est depuis belle lurette - et dans une certaine mesure l'est toujours dans les cas de tous les fleuves internationaux- une source de tensions et de disputes, et un problème de souveraineté, de nécessité stratégique et de fierté nationale. Ces tensions (souvent inextricablement liées à d'autres tensions, et peut-être indissociables de celles-ci) peuvent atteindre un point tel qu'elles déteignent sur les relations géopolitiques entre États au sein d'un bassin et deviennent des obstacles à la croissance en agissant négativement sur l'économie politique régionale et en détournant les ressources du développement économique ${ }^{65} »$. Cher-

64 Claudia Sadoff et David Grey, « Au-delà du fleuve : les avantages de la coopération portant sur les fleuves internationaux ", Banque mondiale, Version provisoire, Document de référence, p.1, in http://iwlearn.net/abt_iwlearn/events/workshops/ouagadougou/readingfiles/worldbank-au-dela-dufleuve.pdf, consulté le 26 août 2013

65 Idem, p.9. 
cher à impliquer les États dans une démarche visant à coopérer autour des ressources qu'ils considèrent comme attribut de souveraineté nationale est de ce fait une entreprise peu commode. La question a d'ailleurs été discutée en 1997 au cours des débats qui ont précédé l'adoption, par l'Assemblée générale des Nations Unies de la Convention sur le droit relatif des cours d'eau internationaux à des fins autres que la navigation.

En effet, les débateurs de la CDI réunis en vue de l'adoption de la convention ont souligné que, lorsqu'un accord particulier est destiné à régir l'ensemble d'un cours d'eau international, il devrait évidemment pouvoir s'appliquer à tous les États de celui-ci, ce qui signifie que tous devraient pouvoir participer aux négociations y relatives et pouvoir en devenir Parties. S'ils s'en désintéressent toutefois, le principe de la relativité des traités s'appliquera, les rapports entre Parties contractantes et États tiers étant alors régis par le droit international général ${ }^{66}$. Si la règle autorisant tous les États du cours d'eau à participer à la négociation et à devenir Parties à l'accord n'a pas été sérieusement contestée, en ce sens qu'elle est exprimée à l'article 4, paragraphe premier de la Convention, Lucius Caflish pense pour sa part que le vrai problème a été celui posé par les accords partiels de cours d'eau, c'est-à-dire ceux conclus par quelques-uns seulement parmi les États concerné et applicables aux seules parties du cours d'eau appartenant à ces États, lorsque ces instruments menacent les droits d'États tiers du même cours d'eau.

À cet égard, l'on se souviendra que l'article 4, paragraphe 2, du projet de la CDI permettait aux États tiers, dans un premier temps, de prendre part aux consultations et négociations relatives à l'accord et, dans une seconde phase, de devenir Parties à celui-ci. La réglementation ainsi proposée soulevait la question de savoir si les pays exclus du cercle des États contractants devraient pouvoir « forcer la porte» de celui-ci.

La RDC n'ayant jamais été à l'origine de l'Accord, peut, se considérer peut-être avec raison, comme un État tiers, qui, au sens de l'article 4 de la convention précitée, a du se contenter du droit de participer à des consultations relatives à un accord devant s'appliquer sur un bassin dont elle abrite $62 \%$ de réserves. Même si par la suite, ce pays s'est engagé dans un mariage forcé en ratifiant l'Accord, l'on ne peut perdre de vue le sentiment souverainiste souvent mis en avant par les États, lors de négociations portant sur de telles matières. Un sentiment général de perte de souveraineté semble animer la conduite de la RDC dans l'application des Accords négociés dans les conditions rappelées. $L$ 'on vérifiera enfin, son attitude face au Code CEMAC/RDC.

\section{b. Le Code CEMAC/RDC : un instrument de la CEMAC}

Le Code CEMAC/RDC a été adopté le même jour que l'Accord créant la CICOS. En effet, la préoccupation majeure de l'Union douanière économique et monétaire (UDEAC), lorsqu'elle s'engage dans un projet d'harmonisation des législations de ses États membres en

66 Caflisch Lucius, « La convention du 21 mai 1997 sur l'utilisation des cours d'eau internationaux à des fins autres que la navigation » In Annuaire français de droit international, volume 43, 1997, p. 774. 
matière de transport fluvial, consiste à faciliter ce transport en adoptant un instrument pour la navigation intérieure commun à tous les États. Il a fallu pour ce faire inclure la RDC qui abrite l'essentiel des voies navigables sur lesquelles devrait s'appliquer le code, cela, sur recommandation de l'UNCEA. Pour y parvenir, l'UDEAC s'appuie sur les accords signés par la Commission tripartite de 1978. A cet effet, les participants ont recommandé au Secrétariat Exécutif de la CEMAC de tout mettre en œuvre pour assurer une large diffusion dudit code en organisant plusieurs séminaires de formation et de sa vulgarisation ${ }^{67}$.

Près de quinze ans depuis sa signature, la RDC qui abrite le plus grand réseau du bassin et qui partage avec la République du Congo et la République Centrafricaine plus de 2.000 $\mathrm{Km}$ des voies transfrontalières fréquentées indistinctement par les bateaux de ces trois pays, ne trouve pas encore intérêt à ratifier le code de la navigation intérieure CEMAC/RDC, contrairement à tous les autres États. Ce qui fait perdurer une insécurité juridique, source de conflit de lois né de la concurrence de deux législations, l'une nationale et l'autre communautaire, devant s'appliquer sur une même voie de navigation. Il est dès lors recommandé d'en examiner le contenu et la portée (2.1) afin de comprendre le regard méfiant de la RDC face à ce document.

\section{aa. Contenu et portée du code CEMAC-RDC}

Le code de la navigation intérieure CEMAC-RDC est un instrument adopté à N'djamena le 17 décembre 1999 en vue d'harmoniser les règles de navigation intérieure dans les pays membres de la CICOS en l'occurrence, la République du Cameroun, la République Centrafricaine, la République démocratique du Congo et la République du Congo. Il classifie les voies de navigation intérieure et détermine les règles et les conditions d'utilisation de ces voies, d'accès aux ports, de protection des voies de navigation, de balisage, d'assistance et de sauvetage. L'article 152 se borne à dire que la navigation sur les voies intérieures donne lieu au payement des droits et taxes fixés, conformément à la législation de chaque État membre. Aussi, l'objectif d'harmonisation des règles se limite ainsi aux seuls aspects techniques liés aux questions de sécurité et aux autres formalités administratives qui ne peuvent réduire les tracasseries et la multiplicité des taxes perçus illégalement par des agents non qualifiés. Près de 15 ans après son adoption, seule la RDC ne l'a pas encore ratifié alors qu'elle est signataire de l'Accord instituant un régime fluvial uniforme créant la CICOS. Elle s'emploie néanmoins à développer d'autres stratégies.

\section{bb. Stratégies développées et raisons invoquées par la RDC pour rejeter le Code CEMAC- $R D C$}

Devant son refus de ratifier le Code CEMAC, la RDC met en œuvre d'autres stratégies, et semble privilégier l'approche bilatérale en s'appuyant sur l'Accord créant la CICOS qui

67 Lire le Communiqué final de la réunion Ministres en charge de la Navigation Intérieure de la CEMAC et de la RDC, Brazzaville, les 05 et 06 Novembre 1999. 
offre la possibilité aux États de se lier dans des Accords spéciaux pour une partie de leur cours d'eau. Dans ce cadre, le pays a amorcé une série de révision de tous les accords qui le lient avec les États voisins. Signée en 1995, la Convention d'exploitation du Pool Malebo ne s'adaptait plus à l'esprit de l'Accord créant la CICOS et au code de la navigation intérieure CEMAC/RDC. Il était de ce fait nécessaire de la réviser. Sous la supervision de la direction juridique et de la réglementation, la réunion des experts de deux États qui ont examiné le projet de révision de la convention d'exploitation, a eu lieu le 11 novembre 2005 à Kinshasa, au siège de la CICOS. En ce sens, la nouvelle Convention d'exploitation du Pool $M_{a l e b o}{ }^{68}$, signée en novembre 2005, semble respecter les dispositions du Code de navigation intérieure $C E M A C / R D C$, en ce qu'elle décrit entre autres les modalités de navigation et les normes minimales pour les unités fluviales exploitant le Pool et engage les États à assurer l'aménagement et l'entretien des domaines publics portuaires. La révision du Protocole d'Accord Tripartite entre la République Centrafricaine, la République du Congo et la RDC relatif à l'entretien des voies navigables d'intérêt commun de 1978 s'inscrit dans la même logique. En vue de rendre conforme ce Protocole au code sans pourtant y adhérer, ce texte a été révisé au cours de la réunion de la commission technique des experts tenue à Brazzaville, les 26 et 27 mai 2008 à cet effet.

Malgré cette attitude de la RDC à contourner le cadre juridique commun à la région, l'importance du réseau navigable pour le reste des États est telle que le retard de la RDC à ratifier le code reste un sujet des préoccupations de la CICOS qui tente de ramener ce pays à marquer son accord. Dans le cadre d'un atelier sur l'appropriation du code tenu du $02 \mathrm{au}$ 03 juillet 2008, les États membres étaient invités à réfléchir sur les facteurs qui pourraient être à la base de ce retard. Au cours des débats, les Experts congolais ayant considéré que le code ne pouvait être appliqué que sur le tronçon commun des voies navigables, ont formulé la déclaration interprétative selon laquelle :

« [... ] L'expression « eaux sous juridiction des États CEMAC/RDC », reprise dans le code notamment à l'article 1, paragraphe 2 doit s'entendre comme « tronçon commun des voies navigables».

Aussi, l'interprétation des textes, la multiplicité des textes, la signalisation et la non uniformité des documents de bord ${ }^{69}$ demeurent les problèmes majeurs à la base de la non application du Code commun par la RDC. Les experts ont de ce fait recommandé au Ministère des Transports et Voies de Communication la constitution d'un Comité de suivi du dossier de la ratification du code ou d'entamer carrément le processus de sa ratification et d'as-

68 Convention d'exploitation du Pool Malebo entre la République du Congo et la République Démocratique du Congo, Kinshasa, CICOS, 22 novembre 2005, [En ligne], [www.cicos.info/siteweb/fil eadmin/documents/Conventions/Convention\%20d'Exploitation\%20du\%20Pool\%20Malebo.pdf] (10 juillet 2013).

69 Rapport de l'atelier national d'appropriation du code de la navigation intérieure CEMAC-RDC tenue au cercle Elaeis du 02 au 03 juillet 2008, disponible en ligne, p5 
sainir le secteur de la navigation intérieure par l'application stricte du décret $\mathrm{n}^{\circ} 036$ déterminant le nombre de services autorisés à œuvrer dans les ports et les postes frontaliers ${ }^{70}$.

\section{CONCLUSION}

L'analyse du cadre juridique sur la navigation intérieure dans le bassin du Congo relève que, ce vaste réseau de transport naturel a de tout temps été un sujet de préoccupation pour les États qui veulent bien tirer profit des conditions naturelles à la circulation des personnes, des biens et services dans une région enclavée. Des efforts de coopération ont été entrepris par les États dès les indépendances malgré les tensions sur fond de revendication de la souveraineté sur tout ou partie du fleuve. Actuellement, ces efforts se poursuivent non sans difficulté au regard des positions souvent prises par la RDC. Même si actuellement ce pays semble réticent quant à son adhésion dans des « instruments élaborés en dehors de son initiative ", il demeure que ses relations de coopération actuelles avec certains riverains ne pourraient présenter aucune contradiction avec des engagements qu'il prendrait dans le cadre multilatéral.

Les décisions de coopération et les niveaux d'engagement pris actuellement dans ses relations avec quelques riverains témoignent une volonté qui pourrait normalement permettre d'élargir la coopération à l'ensemble des espaces sous-régionaux reliés par le bassin du Congo. Une coopération concrète autour du fleuve, de ses affluents, sous affluents et lacs, ouvre, comme nous l'avons démontré, la voie au développement des relations économiques entre sous-régions qui jusqu'à présent, se limitent encore à des accords bilatéraux entre pays des sous-régions différentes ayant une frontière commune. Or de tels accords ne visent que des objectifs limités à certains biefs. Le début d'élargissement de la coopération favorisée actuellement par la mise en place de la CICOS de même que l'existence des structures de coopération comme la CEMAC, la CEEAC, la CEPGL et actuellement la CIRGL, constitue des atouts sur lesquels les États devraient s'appuyer pour élargir la coopération à des États riverains non concernés par le cadre juridique actuel et ceux non riverains du fleuve ayant des interconnexions décrites plus loin.

Au moment même où les sous-régions économiques cherchent à se consolider après un long processus de construction, il est plutôt nécessaire de réfléchir aux rapports qu'elles doivent entretenir, que de repousser une coopération entre celles-ci, tant les découpages actuels de ces sous-régions demeurent artificiels comme le soulignait le professeur Nguyandila. Malgré ces découpages en effet, on le voit bien, certains pays ne voient pas clairement dans quelle sous-région ils doivent entrer, d'autres comme la RDC constatent qu'ils appartiennent objectivement à deux sous-régions différentes. On comprend dès lors que la géographie des sous-régions économiques peut évoluer. Des pays peuvent demeurer en dehors de tout sous-région économique, d'autres peuvent quitter une sous-région, adhérer à deux

70 Rapport de l'atelier national d'appropriation du code de la navigation intérieure CEMAC-RDC, op.cit. 
sous-régions ou changer de sous-région, en fonction des intérêts en présence et de l'évolution politique, linguistique et économique du continent.

Par contre, la géographie d'un bassin fluvial ne change pas, les lois physiques qui régissent son comportement ne varient pas. Les intérêts des États riverains par rapport aux ressources du bassin y compris pour la navigation, sont permanents, le fleuve étant un lien naturel. Dans les États membres de la CICOS par contre, le transport fluvial n'a pratiquement pas connu d'évolution depuis l'adoption des politiques de libéralisation observées dans ce sous-secteur. Le cabotage joue encore un rôle très marginal dans l'intégration physique de la sous région malgré ses importantes voies d'eau navigables.

Dans l'affaire Gabčíkovo-Nagymaros, la Cour internationale de justice a mis en exergue le fondement juridique et l'importance potentielle des approches de gestion commune d'un cours d'eau international, en s'appuyant sur un passage de l'affaire relative à l'Oder :

"La communauté d'intérêts sur un fleuve navigable devient la base d'une communauté de droit, dont les traits essentiels sont la parfaite égalité de tous les États riverains dans l'usage de tout le parcours du fleuve et l'exclusion de tout privilège d'un riverain quelconque par rapport aux autres. "

Bien que de très nombreux analystes aient évoqué le principe d'une communauté d'intérêts et le recours correspondant à une approche commune de gestion des fleuves internationaux, rares sont ceux qui prétendraient qu'une telle approche s'est transformée, ou a des chances de se transformer bientôt, en une obligation du droit international général ou coutumier. D'autres auteurs, qui s'appuient sur l'expérience européenne, notent par contre que «la gestion des cours d'eau internationaux par les États de l'Europe du Nord repose clairement sur la théorie de la gestion commune impliquant des organes de gestion mixtes - commissions des cours d'eau frontaliers - qui réglementent toutes les utilisations des cours d'eau internationaux dans la région $~^{71}$; d'autres encore attirent l'attention sur le coût de la

71 Programme des Nations Unies pour l'environnement, PNUE, Amélioration de la gouvernance des eaux transfrontalières grâce à la gestion intégrée des ressources en eau, Document de synthèse, $p$. 15; Jean Marc Thouvenin, «Droit international général des utilisations des fleuves internationaux», in Bogdan Aurescu, Alain Pellet (dir.), Actualité du droit des fleuves internationaux, Actes des journées d'études du 24 et 25 octobre 2008, Paris Pedone, 2010, pp.107-128; Christina Leb, «Pluralité d'utilisation et gestion commune intégrée», in Bogdan Aurescu, Alain Pellet, op.cit, pp. 129-138; Igor Grexa, « La gestion commune des fleuves internationaux. L'exemple de la convention sur le Danube de 1948», in Bogdan Aurescu, Alain Pellet, idem, pp.139-147. 
non-coopération en matière de gestion des cours d'eau internationaux ${ }^{72}$ quelque soit la forme d'usage.

Aussi, dans un contexte international actuel, la RDC aura plus d'intérêt à développer et à élargir sa coopération avec l'ensemble des États riverains au bassin du fleuve Congo. Car, si la navigation est le seul aspect analysé dans le cadre de cette étude, elle n'est pas le seul à pouvoir intéresser les riverains. Les questions plus dramatiques tels que le partage des quotas d'eau, de pêche entre riverains ou celles de transfert d'eau interbassin dans des régions déjà visiblement menacées par les impacts des changements climatiques pourraient, demain exacerber les conflits s'ils ne peuvent s'appuyer sur une architecture juridique et institutionnelle déjà expérimentée et développée en matière de navigation.

S'étant déjà engagée dans la CICOS, la RDC devra considérer sa position dans le bassin et au sein de cette organisation et se montrer dès lors plus apte à prendre des initiatives qui favorisent la coopération avec les autres riverains et plus continuer à attendre d'être invitée dans des négociations qui aboutissent à la mise sur pied des mécanismes de gestion commune dont elle n'appliquera pas le cadre juridique accepté.

\section{BIBLIOGRAPHIE}

\section{TEXTES OFFICIELS}

\section{A. Textes internationaux}

Accord instituant un régime fluvial uniforme et créant la CICOS, Brazzaville, 6 novembre 1999 Acte constitutif de l'Union africaine, Lomé, juillet, 2000

Acte général de la Conférence de Berlin, février 1885, in www.congoforum.be/upldocs/Acte\%20de\%2

0Berlin\%201885.pdf, consulté le 22 avril 2013

Code de la navigation intérieure CEMAC-RDC, N'djamena, 17 décembre 1999.

72 Claudia Sadoff et David Grey, Au-delà du fleuve : les avantages de la coopération portant sur les fleuves internationaux, version provisoire, Banque mondiale, pp.9-12 ; Rapport mondial sur le développement humain, 2006, chapitre 6, la gestion des eaux transfrontalières, pp.209-214 ; Fréderic Lassere et Annabelle Bouet, «Le droit international réglera-t-il les litiges du partage de l'eau ? Le bassin du Nil et quelques autres cas, Études internationales, vol.33, n³, 2002, p.497-514 ; Laurence Boisson de Chazournes, Le règlement des différends internationaux relatifs à l'eau, Études et documents du Conseil d'État, pp.489-513 ; Vanessa Richard, Gestion privée de l'eau : où va le droit international ?, Études et documents du Conseil d'État, pp.515-524; Javier SOLANA MADARIAGA, Changements climatiques et sécurité internationale, Document établi par le haut Représentant et la Commission européenne à l'attention du Conseil européen, in www.europa.eu ; Abdessamad DRIS, L'eau matière stratégique et enjeu de sécurité au $21^{\text {ème }}$ siècle, Mémoire de DEA, Université Paris 10, Sciences politiques, 2005 ; EAGLES, East African Great Lake Ecosystem Sensitivity to changes, in

http://www.eagles-kivu.be/, consulté le 26 août 2013. 
Communiqué final de la réunion Ministres en charge de la Navigation Intérieure de la CEMAC et de la RDC, Brazzaville, les 05 et 06 Novembre 1999.

Convention de Saint-Germain-en-Laye, du 10 septembre 1919, Revue juridique et politique (Paris), X1Xth year, No. 2 (April- June 1965).

Convention de Genève du 9 décembre 1923 relative à l'aménagement des forces hydrauliques intéressant plusieurs États, Société des Nations, Recueil des traités, vol. 36.

Convention des Nations Unies sur le droit relatif aux utilisations des cours d'eau à des fins autres que la navigation, New York, le 21 mai 1997, Résolution 51/229 de 1'Assemblée générale, annexe, Documents officiels de l'Assemblée générale, cinquante et unième session, Supplément $\mathbf{n}^{\circ} 49$ (A/51/49), pp.286-305.

Convention d'exploitation du Pool Malebo entre la République du Congo et la République Démocratique du Congo, Kinshasa, CICOS, 22 novembre 2005, [En ligne], [www.cicos.info/siteweb/fileadmin/documents/Conventions/Convention\%20d'Exploitation\%20du\%20Pool\%20Malebo.pdf] (10 juillet 2013).

Protocole d'accord tripartite relatif aux modalités d'entretien des voies navigables d'intérêt commun entre la République Centrafricaine, la République du Congo et la République Démocratique du Congo relatif à l'entretien des voies navigables d'intérêt commun de 1978.

\section{B. Textes nationaux}

Loi $\mathrm{n}^{\circ}$ 74-026 du 2 décembre 1974 portant création de la Compagnie maritime zaïroise, J.O.Z., $\mathrm{n}^{\circ} 4,15$ février 1975, p. 124.

Décret $\mathrm{n}^{\circ} 0051$ du 7 novembre 1995portant création et statuts d'une entreprise publique dénommée l'Office national des transports, en abrégé «O.N.A.T.R.A.», in Code Larcier, Tome III, vol. 1, Édition 2002.

Ordonnance 80-256 du 2 novembre 1980 portant création et statuts de l'Office de gestion du fret maritime, J.O.Z., n²2, 15 novembre 1980.

Ordonnance $\mathrm{N}^{\circ} 71-308$ du 3 décembre 1971 établissant le cahier des charges de la Régie des voies fluviales.

Ordonnance n78-198 du 5 mai 1978 portant statuts d'une entreprise publique dénommée Régie des voies maritimes, J.O.Z., n¹0, 15 mai 1978, Code Larcier, Tome III, vol. 1, Édition 2002, p.362

\section{OUVRAGES, MÉMOIRES, ARTICLES ET AUTRES DOCUMENTS}

Abdessamad DRIS, L'eau matière stratégique et enjeu de sécurité au 21ème siècle, mémoire de DEA, Université Paris 10, Sciences politiques, 2005.

Alain Pellet, « Conclusions générales », Société Française pour le droit international, L'eau en droit international, Colloque d'Orléans, Paris, Pedone, 2011, pp. 395-402.

Anonyme, Défis et opportunités liés à l'eau 3, s.d, pp.37-122.

Anonyme, Thématique, section 30, L'hydro politique et les relations internationales http://www.congre safsp2009.fr/sectionsthematiques/st30/st30julien.pdf, le 26 août 2013. 
Biyoya Makutu Kahandja, $(\mathrm{PH})$ «Les guerres transnationales de la région des grands lacs de l'Afrique centrale : enjeux et défis », in Joseph Vincent Ntunda Ebodé, in Piraterie et terrorisme : de nouveaux défis sécuritaires en Afrique centrale, Friedrich Ebert Stiftung, Presses Universitaires d'Afrique, Yaoundé, Cameroun, (FES) pp.185-199.

Bruno Hellendorff, « L'eau, les conflits et la coopération Gestion de l'eau en Afrique de l'Ouest: Risques et opportunités 》, in www.grip.org/sites/grip.../NA_2013-02-08_fr_B-HELLENDORFF.pdf consulté le 13 février 2013.

Caflisch Lucius, «La convention du 21 mai 1997 sur l'utilisation des cours d'eau internationaux à des fins autres que la navigation », Annuaire français de droit international, volume 43, 1997, pp. 751-798.

Cécile Pilarski, La gestion partagée des ressources en eau dans le bassin du Congo : état des lieux et perspectives, mémoire présenté à la Faculté des études supérieures de l'Université Laval, 2009.

Cemac, Présentation de la CEMAC, [En ligne], [www.cemac.cf/presentationCEMAC.htm]

Charles Rousseau, Précis de droit international public approfondi, Dalloz, Paris, 1975.

Christina Leb, «Pluralité d'utilisation et gestion commune intégrée», in Bogdan Aurescu, Alain Pellet (dir.), Actualité du droit des fleuves internationaux, Actes des journées d'études du 24 et 25 octobre 2008, Paris, Pedone, 2010, pp.129-138.

$\mathrm{Cicos} / \mathrm{Gtz}$, Plan d'action stratégique pour la promotion de la navigation dans le bassin Congo-Oubangui-Sangha, Kinshasa, CICOS, 2007 p. 1 (ci-après PAS navigation).

Claudia Sadoff, David Grey, Au-delà du fleuve : les avantages de la coopération portant sur les fleuves internationaux, Banque mondiale, version provisoire, in http://iwlearn.net/abt_iwlearn/events/work shops/ouagadougou/readingfiles/worldbank-au-dela-du-fleuve.pdf, le 26 août 2013.

Claudia W. Sadoff, David Grey, Les fleuves internationaux d'Afrique Une perspective économique, Banque Mondiale, http://earthmind.net/rivers/docs/worldbank-fleuve-internationaux-d'Afri que.pdf, consulté le 26 août 2013.

Commission internationale du bassin Congo-Oubangui-sangha « CICOS », Rapport de l'atelier national d'appropriation du code de la navigation intérieure CEMAC-RDC tenue au cercle Elaeis du 02 au 03 juillet 2008, disponible en ligne ; Rapport de la réunion de la commission technique des experts sur la révision du protocole d'accord tripartite RCA-RC-RDC relatif aux modalités d'entretien des voies navigables d'intérêt commun, Brazzaville, les 26 et 27 mai 2008 ; Rapport d'activités, 2005.

Conférence Internationale pour la région des Grands Lacs (CIRGL), Étude de faisabilité sur la réhabilitation et la navigabilité du fleuve Congo, Octobre 2005 (Rev. 30 août 06).

David Ruzié, Droit international public, $15^{\text {ème }}$ édition, Dalloz, 2000, pp.160-161.

Frédéric Julien, «L'eau dans les relations internationales: la guerre ou la paix? D’un déterminisme à l'autre », (Université d'Ottawa), Congrès AFSP 2009.

Frédéric Lasserre, Annabelle Bouet, « Le droit international réglera-t-il les litiges du partage de l'eau? Le bassin du Nil et quelques autres cas », Études internationales, vol.33, n³, 2002, pp.497-514.

Hellendorf Bruno, "L'eau, les conflits et la coopération. Gestion de l'eau en Afrique de l'Ouest : Risques et opportunités », Note d'Analyse du GRIP, 2013, Bruxelles, http://grip.org/fr/node/797, consulté le 30 juillet 2013 . 
Igor Grexa, « La gestion commune des fleuves internationaux. L'exemple de la convention sur le Danube de 1948», in Bogdan Aurescu, Alain Pellet (dir.), Actualité du droit des fleuves internationaux, Actes des journées d'études du 24 et 25 octobre 2008, Paris, Pedone, 2010, pp.139-147.

Institut de droit international, point II/4 de la Résolution sur les usages des cours d'eau internationaux, Annuaire de l'Institut de droit international 1911, vol. 24.

Javier Solana madariaga, Changements climatiques et sécurité internationale, Document établi par le haut Représentant et la Commission européenne à l'attention du Conseil européen, in www.europa .eu.

Jean Grosdidier de Matons, Un recueil des instruments juridiques internationaux, Programme de politiques de transport en Afrique subsaharienne Document de travail SSATP N 73F, mai 2004.

Jean Marc Thouvenin, «Droit international général des utilisations des fleuves internationaux», in Bogdan Aurescu, Alain Pellet (dir.), Actualité du droit des fleuves internationaux, Actes des journées d'études du 24 et 25 octobre 2008, Paris, Pedone, 2010, pp.107-128;

Jochen Sohnlel, « Le dispositif juridique de l'Europe pour appréhender les conflits transfrontaliers sur l'eau », Lex Electronica, vol. 12 n², 2007.

Laurence Boisson de Chazournes, "Le règlement des différends internationaux relatifs à l'eau ", Études et documents du Conseil d'État, pp.489-513.

Mara Tignino, «L'eau et son rôle dans la paix et la sécurité internationales», www.icrc.org/fre/assets/fil es/other/irrc-879-tignino-fre.pdf.

\section{Mubiala Mutoy, L'évolution du droit des cours d'eau internationaux à la lumière de l'expérience africaine, notamment dans le bassin du Congo/Zaïre. Presses universitaires de France, Paris, 1995.}

Nguyandila Malengana (C), Droit international public, domaine public international, cours, faculté de droit, Université de Kinshasa, 2006; Indépendance de la République démocratique du Congo et les engagements internationaux antérieurs, publications de l'Université de Kinshasa, 1971; Document manuscrit, inédit

Ngyuen Quoc Dinh, Patrick Daillier, Matthias Forteau, Alain Pellet, Droit international public, $8^{\text {ème }}$ édition, L.G.D.J, Paris, 2009.

Programme des Nations unies pour l'environnement, (PNUE), Amélioration de la gouvernance des eaux transfrontalières grâce à la gestion intégrée des ressources en eau, Document de synthèse Document de synthèse; Rapport mondial sur le développement humain, 2006, chapitre 6, la gestion des eaux transfrontalières, pp.203-231.

Robert Arnould, «La canalisation du fleuve Congo à l'aval de Kinshasa : un défi pour le XXIème siècle», Bulletin de la Société géographique de Liège, n 46, 2005, pp.99-117.

Sylvie AYIMPAM, «Vie matérielle, échanges et capitalisme sur la rive méridionale du Pool du fleuve Congo 1815-1930 », Collection Clio en Afrique, № 18, Edition du Centre d’Études des Mondes Africains, Ex-en-Province, 2006.

Union africaine, Étude sur le Programme de Développement des Infrastructures en Afrique (PIDA), Rapport de la Phase 1, Provisoire, mars 2011. 
Vanessa Richard, « Gestion privée de l'eau: où va le droit international? », Études et documents du Conseil d'État, pp.515-524. 